\title{
Risk Pricing over Alternative Investment Horizons *
}

\author{
Lars Peter Hansen \\ University of Chicago and the NBER
}

June 19, 2012

\begin{abstract}
I explore methods that characterize model-based valuation of stochastically growing cash flows. Following previous research, I use stochastic discount factors as a convenient device to depict asset values. I extend that literature by focusing on the impact of compounding these discount factors over alternative investment horizons. In modeling cash flows, I also incorporate stochastic growth factors. I explore dynamic value decomposition (DVD) methods that capture concurrent compounding of a stochastic growth and discount factors in determining risk-adjusted values. These methods are supported by factorizations that extract martingale components of stochastic growth and discount factors, These components reveal which ingredients of a model have long-term implications for valuation. The resulting martingales imply convenient changes in measure that are distinct from those used in mathematical finance, and they provide the foundations for analyzing model-based implications for the term structure of risk prices. As an illustration of the methods, I re-examine some recent preference based models. I also use the martingale extraction to revisit the value implications of some benchmark models with market restrictions and heterogenous consumers.
\end{abstract}

*I thank Rui Cui, Mark Hendricks, Eric Renault, Grace Tsiang and especially Fernando Alvarez for helpful discussions in preparing this chapter. 


\section{Introduction}

Model-based asset prices are represented conveniently using stochastic discount factors. These discount factors are stochastic in order that they simultaneously discount the future and adjust for risk. Hansen and Richard (1987), Hansen and Jagannathan (1991), Cochrane (2001) and Singleton (2006) and show how to construct and use stochastic discount factors to compare implications of alternative asset pricing models.

This chapter explores three interrelated topics using stochastic discount factors. First I explore the impact of compounding stochastic discount factors over alternative investment horizons required for pricing asset payoff over multi-period investment horizons. The impact of compounding with state dependent discounting is challenging to characterize outside the realm of log-normal models. I discuss methods that push beyond log-linear approximations to understand better valuation differences across models over alternative investment horizons. They allow for nonlinearities in the underlying stochastic evolution of the economy. As an important component to my discussion, I show how to use explicit models of valuation to extract the implications that are durable over long-horizons by deconstructing stochastic discount factors in revealing ways.

State dependence in the growth of cash flows provides a second source of compounding. Second, I explore ways to characterize the pricing of growth rate risk by featuring the interaction between state dependence in discounting and growth. To support this aim I revisit the study of holding-period returns to cash flows over alternative investment horizons, and I suggest a characterization of the "term-structure of risk prices" embedded in the valuation of cash flows with uncertain growth prospects. I obtain this second characterization by constructing elasticities that show how expected returns over different investment horizons respond to changes in risk exposures. Risk premia reflect both the exposure to risk and the price of that exposure. I suggest ways to quantify both of these channels of influence. In particular, I extend the concept of risk prices used to represent risk-return tradeoffs to study multi-period pricing and give a more complete understanding of alternative structural models of asset prices. By pricing the exposures of the shocks to the underlying macroeconomy, I provide a valuation counterparts to impulse response functions used extensively in empirical macroeconomics.

In addition to presenting these tools, I also explore ways to compare explicit economic models of valuation. I consider models with varied specifications of investor preferences and beliefs including models with habit persistent preferences, recursive utility preferences for 
which the intertemporal composition of risk matters, preferences that capture ambiguity aversion and concerns for model misspecification. I also explore how the dynamics of cross-sectional distribution of consumption influence valuation when complete risk sharing through asset markets is not possible. I consider market structures that acknowledge private information among investors or allow for limited commitment. I also consider structures that allow for solvency constraints and the preclusion of financial market contracting over idiosyncratic shocks.

The remainder of this chapter is organized as follows. In section 2 I suggest some valuable characterizations of stochastic discount factor dynamics. I accomplish this in part by building a change of measure based on long-term valuation considerations in contrast to the familiar local risk-neutral change of measure. In section 3 I extend the analysis by introducing a stochastic growth functional into the analysis. This allows for the interaction between stochastic components to discounting and growth over alternative investment or payoff horizons. I illustrate the resulting dynamic value decomposition (DVD) methods using some illustrative economies that feature the impact of investor preferences on asset pricing. Finally in section 4, I consider some benchmark models with frictions to assess which frictions have only short-term consequences for valuation. 


\section{Stochastic discount factor dynamics}

In this section we pose a tractable specification for stochastic discount factor dynamics that includes many of the parametric specifications in the literature. I then describe methods that characterize the implied long-term contributions to valuation and explore methods that help us characterize impact of compounding stochastic discount factors over multiple investment horizons.

\subsection{Basic setup}

I begin with an information set $\mathcal{F}_{0}$ (sigma algebra) two random vectors: $Y_{0}$ and $X_{0}$ that are $\mathcal{F}_{0}$ measurable. I consider an underlying stochastic process $(Y, X)=\left\{\left(Y_{t}, X_{t}\right): t=0,1, \ldots\right\}$ and use this process to define an increasing sequence of information sets (a filtration) $\left\{\mathcal{F}_{t}: t=0,1, \ldots\right\}$ where $\left(Y_{u}, X_{u}\right)$ is measurable with respect to $\mathcal{F}_{t}$ for $0 \leq u \leq t$. Following Hansen and Scheinkman (2012b), I assume a recursive structure to the underlying stochastic process:

Assumption 2.1. The conditional distribution $\left(Y_{t+1}-Y_{t}, X_{t+1}\right)$ conditioned on $\mathcal{F}_{t}$ depends only on $X_{t}$ and is time invariant.

It follows from this assumption that $Y$ does not "Granger cause" $X$, that $X$ is itself a Markov process and that $\left\{Y_{t+1}-Y_{t}\right\}$ is a sequence of independent and identically distributed random vectors conditioned on the entire $X$ process. $^{1}$

I suppose that the processes that we use in representing asset values have a recursive structure.

Definition 2.2. An additive functional is a process whose first-difference has the form:

$$
A_{t+1}-A_{t}=\kappa\left(Y_{t+1}-Y_{t}, X_{t+1}\right)
$$

It will often be convenient to initialize the additive functional: $A_{0}=0$, but we allow for other initial conditions as well. I model stochastic growth and discounting using additive functionals after taking logarithms. This specification is flexible enough to include many commonly-used time series models. I relate the first-difference of $A$ to the first-difference of $Y$ in order to allow the increment in $A$ to depend on the increment in $Y$ in continuous-time counterparts.

\footnotetext{
${ }^{1}$ For instance, see Bickel et al. (1998). I may think of this conditional independence as being more restrictive counterpart to Sims (1972)'s alternative characterization of Granger (1969) causality.
} 


\subsection{A convenient factorization}

Let $S_{t}$ denote the stochastic discount factor between dates zero and $t$. The implicit discounting over a single time period between $t$ and $t+1$ is embedded in this specification

and is given by ratio $\frac{S_{t+1}}{S_{t}}$. The discounting is stochastic to accommodate risk adjustments in valuation. In representative consumer models with power utility functions

$$
\frac{S_{t+1}}{S_{t}}=\exp (-\delta)\left(\frac{C_{t+1}}{C_{t}}\right)^{-\rho}
$$

where $C_{t}$ is aggregate consumption at date $t, \delta$ is the subjective rate of discount, and $\frac{1}{\rho}$ is the elasticity of intertemporal substitution. The formula on the right-hand side of (1) is the one-period intertemporal marginal rate of substitution for the representative consumer. This particular formulation is very special and problematic from an empirical perspective, but I will still use it as revealing benchmark for comparison.

One-period stochastic discount factors have been used extensively to characterize the empirical support, or lack thereof, for understanding one-period risk return tradeoffs. My aim, however, to explore valuation for alternative investment horizons. For instance, to study the valuation of date $t+2$ payoffs from the vantage point of date $t$, I am lead to compound two one-period stochastic discount factors:

$$
\left(\frac{S_{t+2}}{S_{t+1}}\right)\left(\frac{S_{t+1}}{S_{t}}\right)=\frac{S_{t+2}}{S_{t}}
$$

Extending this logic leads me to the study of the stochastic discount factor process $S$, which embeds the stochastic discounting for the full array of investment horizons.

Alvarez and Jermann (2005), Hansen et al. (2008), Hansen and Scheinkman (2009) and Hansen (2012) suggest, motivate and formally defend a factorization of the form:

$$
\frac{S_{t+1}}{S_{t}}=\exp (-\eta)\left(\frac{M_{t+1}}{M_{t}}\right)\left[\frac{f\left(X_{t+1}\right)}{f\left(X_{t}\right)}\right]
$$

where $M$ is a martingale and $X$ is a Markov process. I will show subsequently how to construct $f$. I will give myself flexibility in how I normalize $S_{0}$. While sometimes I will set it to one, any strictly positive normalization will suffice. In what follows we suppose that both $\log S$ and $\log M$ are additive functionals. Extending this formula to multiple 
investment horizons:

$$
\frac{S_{t}}{S_{0}}=\exp (-\eta t)\left(\frac{M_{t}}{M_{0}}\right)\left[\frac{f\left(X_{t}\right)}{f\left(X_{0}\right)}\right]
$$

There are three components to the this factorization, terms that I will interpret after I supply some more structure. Notice that each of the logarithms of each of the three components are themselves additive functionals.

I construct factorization (2) by solving the Perron-Frobenius problem:

$$
E\left[\left(\frac{S_{t+1}}{S_{t}}\right) e\left(X_{t+1}\right) \mid X_{t}=x\right]=\exp (-\eta) e(x)
$$

where $e$ is a positive function of the Markov state. Then

$$
\frac{M_{t}}{M_{0}}=\exp (\eta t)\left(\frac{S_{t}}{S_{0}}\right)\left[\frac{e\left(X_{t}\right)}{e\left(X_{0}\right)}\right]
$$

is a martingale. Inverting this relation: gives (3) with $f=\frac{1}{e}$.

The preceding construction is not guaranteed to be unique. See Hansen and Scheinkman (2009) and Hansen (2012) for discussions. Recall that positive martingales with unit expectations can be used to induce alternative probability measures via a formula

$$
E\left(M_{t} \psi_{t} \mid \mathcal{F}_{0}\right)=\widetilde{E}\left(\psi_{t} \mid \mathcal{F}_{t}\right)
$$

for any bounded $\psi_{t}$ that is in the date $t$ information set (is $\mathcal{F}_{t}$ measurable). It is straightforward to show that under this change-of-measure, the process $X$ remains Markov and that Assumption 2.1 continue to hold. This martingale construction is not guaranteed to be unique, however. There is at most one such construction for which the martingale $M$ induces stochastically stable dynamics where stochastic stability requires:

Assumption 2.3. Under the change of probability measure,

$$
\lim _{t \rightarrow \infty} \widetilde{E}\left[\phi\left(Y_{t}-Y_{t-1}, X_{t}\right) \mid X_{0}=x\right]=\widetilde{E}\left[\phi\left(Y_{t}-Y_{t-1}, X_{t}\right)\right]
$$

for any bounded Borel measurable function $\phi$. The expectation on the right-hand side uses a stationary distribution implied by the change in the transition distribution. ${ }^{2}$

\footnotetext{
${ }^{2}$ One way to characterize the stationary distribution is to solve $E\left[\psi\left(X_{0}\right) M_{0}\right]=$ $E\left(\widetilde{E}\left[\psi\left(X_{1}\right) \mid X_{0}=x\right] M_{0}\right)$.
} 
See Hansen and Scheinkman (2009) and Hansen (2012) for discussions. There is a well developed set of tools for analyzing Markov processes that can be leveraged to check this restriction. See Meyn and Tweedie (1993) for an extensive discussion of these methods.

The version of factorization (2) that preserves this stochastic stability is of interest for the following reason. It allow me to compute:

$$
E\left[S_{t} \phi\left(Y_{t}-Y_{t-1}, X_{t}\right) \mid X_{0}=x\right]=\exp (-\eta t) e(x) \widetilde{E}\left[\frac{\phi\left(Y_{t}-Y_{t-1}, X_{t}\right)}{e\left(X_{t}\right)} \mid X_{0}=x\right]
$$

Under stochastic stability,

$$
\begin{aligned}
\lim _{t \rightarrow \infty} \frac{1}{t} \log E\left[S_{t} \phi\left(Y_{t}-Y_{t-1}, X_{t}\right) \mid X_{0}=x\right] & =-\eta \\
\lim _{t \rightarrow \infty} \log E\left[S_{t} \phi\left(Y_{t}-Y_{t-1}, X_{t}\right) \mid X_{0}=x\right]+\eta t & =\log e(x)+\log \widetilde{E}\left[\frac{\phi\left(Y_{t}-Y_{t-1}, X_{t}\right)}{e\left(X_{t}\right)}\right]
\end{aligned}
$$

provided that $\phi>0$. Thus the change-in-probability absorbs the martingale component to stochastic discount factors. The rate $\eta$ is the long-term interest rate, which is evident from (5) when we set $\phi$ to be a function that is identically one. ${ }^{3}$

\subsection{Other familiar changes in measure}

In the pricing of derivative claims, researchers often find it convenient to use the so called "risk neutral" measure. To construct this in discrete time, form

$$
\frac{M_{t+1}}{M_{t}}=\frac{S_{t+1}}{E\left(S_{t+1} \mid \mathcal{F}_{t}\right)}
$$

Then $M$ is a martingale with expectation equal to one provided that $E M_{0}=1$. An alternative stochastic discount factor is:

$$
\frac{S_{t+1}}{S_{t}}=\left(\frac{M_{t+1}}{M_{t}}\right) E\left(\frac{S_{t+1}}{S_{t}} \mid \mathcal{F}_{t}\right) .
$$

\footnotetext{
${ }^{3}$ I have added sufficient structure as to provide a degenerate version of the Dybvig et al. (1996) characterization of long-term rates. Dybvig et al. (1996) argue that long-term rates should be weakly increasing.
} 
The risk-neutral probability is the probability measure associated with the martingale $M$, and the one-period interest rate on a discount bond is:

$$
-\log E\left(\frac{S_{t+1}}{S_{t}} \mid \mathcal{F}_{t}\right) .
$$

Absorbing the martingale into the change of measure, the one period prices are compute by discounting using the riskless rate, justifying the term "risk-neutral measure." Whenever the one-period interest rate is state independent, it is equal to $\eta$; and factorizations (2) and (6) coincide with $e=f=1$ (or some other positive constant).

When interest rates are expected to vary over time, this variation in effect gives an adjustment for risk over multiple investment horizons. An alternative would be to use a different change of measure for each investment horizon, but this is not very convenient conceptually. ${ }^{4}$ Instead I find it preferable to use a single change of measure with a constant adjustment to the long-term decay rate $\eta$ in the stochastic discount factor that is state independent as in (2).

\subsection{Log-linear models}

It is commonplace to extract permanent shocks as increments in martingale components of time series. This approach is related but distinct from the approach that I have sketched. The connection is closest when the underlying model of a stochastic discount factor is log-linear with normal shocks. See Alvarez and Jermann (2005) and Hansen et al. (2008). Suppose that

$$
\begin{aligned}
\log S_{t+1}-\log S_{t} & =-\mu+H \cdot X_{t}+G \cdot W_{t+1} \\
X_{t+1} & =A X_{t}+B W_{t+1}
\end{aligned}
$$

where $W$ is a multivariate sequence of standard normally distributed random vectors with mean zero and covariance $I$ and $A$ is a matrix with stable eigenvalues (eigenvalues with absolute values that are strictly less than one). In this case we can construct a martingale component $m$ in logarithms and

$$
\log S_{t}-\log S_{0}=-\nu t+m_{t}-m_{0}+\mathrm{f} \cdot X_{t}-\mathrm{h} \cdot X_{0}
$$

\footnotetext{
${ }^{4}$ Such changes in measure are sometimes called forward measures. See Jamshidian (1989) for an initial application of these measures.
} 
where $m$ is a an additive martingale satisfying:

$$
m_{t+1}-m_{t}=\left[G^{\prime}+H^{\prime}(I-A)^{-1} B\right] W_{t+1},
$$

and

$$
\mathrm{f} \cdot X_{t}=-H^{\prime}(I-A)^{-1} X_{t}
$$

Increments to the additive martingale are permanent shocks, and shocks that are uncorrelated have only transient consequences.

While $m$ is an additive martingale, $\exp (m)$ is not a martingale. It is straightforward to construct the martingale $M$ by forming

$$
\frac{M_{t}}{M_{0}}=\exp \left(m_{t}-m_{0}\right) \exp \left[-\frac{t}{2}\left|G^{\prime}+H^{\prime}(I-A)^{-1} B\right|^{2}\right]
$$

where the second term adjusts is a familiar log-normal adjustment. With stochastic volatility models or regime-shift models, the construction is not as direct. See Hansen (2012) for a discussion of a more general link be between martingale constructions for additive processes and factorization $(3) .^{5}$

\subsection{Model-based factorizations}

Factorization (3) provides a way to formalize long-term contributions to valuation. Consider two alternative stochastic discount factor processes, $S$ and $S^{*}$ associated with two different models of valuation.

Definition 2.4. The valuation implications between model $S$ and $S^{*}$ are transient if these processes share a common value of the long-term interest rate $\eta$ and the martingale component $M$.

Consider the factorization (3) for the power utility model mentioned previously:

$$
S_{t}^{*}=\exp (-\delta t)\left(\frac{C_{t}}{C_{0}}\right)^{-\rho}=\exp \left(-\eta^{*} t\right)\left(\frac{M_{t}^{*}}{M_{0}^{*}}\right)\left[\frac{f^{*}\left(X_{t}\right)}{f^{*}\left(X_{0}\right)}\right]
$$

where $\delta$ is the subjective rate of discount, $\rho>0$, and $\left(\frac{C_{t}}{C_{0}}\right)^{-\rho}$ is the (common) intertemporal

\footnotetext{
${ }^{5}$ The martingale extraction in logarithms applies to a much larger class of processes and results in an additive functional. The exponential of the resulting martingale shares a martingale component in the level factorization (3) with the original process.
} 
marginal rate of substitution of an investor between dates zero and $t$. I assume that $\log C$ satisfies Assumption 2.1. It follows immediately the the logarithm of the marginal utility process, $\gamma \log C$ satisfies this same restriction. In addition the function $f^{*}=\frac{1}{e^{*}}$ and $\left(e^{*}, \eta^{*}\right)$ solves the eigenvalue equation (4) including the imposition of stochastic stability.

Suppose for the moment we hold fixed the consumption process as a device to understand the implications of changing preferences. Bansal and Lehmann (1997) noted that the stochastic discount factors for many asset pricing models have a common structure. I elaborate below. The one-period ratio of the stochastic discount factor is:

$$
\frac{S_{t+1}}{S_{t}}=\left(\frac{S_{t+1}^{*}}{S_{t}^{*}}\right)\left[\frac{h\left(X_{t+1}\right)}{h\left(X_{t}\right)}\right]
$$

From this baseline factorization,

$$
\frac{S_{t}}{S_{0}}=\exp (-\eta t)\left(\frac{M_{t}}{M_{0}}\right)\left[\frac{f\left(X_{t}\right) h\left(X_{t}\right)}{f\left(X_{0}\right) h\left(X_{0}\right)}\right] .
$$

The counterpart for the eigenfunction $e$ is $\frac{1}{f^{*} h}$. Thus when factorization (8) is satisfied, the long-term interest rate $\eta$ and the martingale component to the stochastic discount factor are the same as those with power utility. The function $h$ contributes "transient" components to valuation. Of course these transient components could be highly persistent.

While my aim is to provide a more full characterization of the impact of the payoff horizon on the compensation for exposure to risk, locating permanent components to models of valuation provides a good starting point. It is valuable to know when changes in modeling ingredients has long-term consequences for valuation and when these changes are more transient in nature. It is also valuable to understand when "transient changes" in valuation persist over long investment horizons even though the consequences eventually vanish. The classification using martingale components is merely an initial step for a more complete understanding.

I now explore the valuation implications of some alternative specifications of investor preferences.

\subsubsection{Consumption externalities and habit persistence}

See Abel (1990), Campbell and Cochrane (1999), Menzly et al. (2004) and Garcia et al. (2006) for representations of stochastic discount factors in the form (8) for models with history dependent measures of consumption externalities. A related class of models are 
those in which there are intertemporal complementaries in preferences of the the type suggested by Sundaresan (1989), Constantinides (1990) and Heaton (1995). As argued by Hansen et al. (2008) these models also imply stochastic discount factors that can be expressed as in (8).

\subsubsection{Recursive utility}

Consider a discrete-time specification of recursive preferences of the type suggested by Kreps and Porteus (1978) and Epstein and Zin (1989). I use the homogeneous-of-degreeone aggregator specified in terms of current period consumption $C_{t}$ and the continuation value $V_{t}$ for prospective consumption plan from date $t$ forwards:

$$
V_{t}=\left[\left(\zeta C_{t}\right)^{1-\rho}+\exp (-\delta)\left[\mathcal{R}_{t}\left(V_{t+1}\right)\right]^{1-\rho}\right]^{\frac{1}{1-\rho}}
$$

where

$$
\mathcal{R}_{t}\left(V_{t+1}\right)=\left(E\left[\left(V_{t+1}\right)^{1-\gamma} \mid \mathcal{F}_{t}\right]\right)^{\frac{1}{1-\gamma}}
$$

adjusts the continuation value $V_{t+1}$ for risk. With these preferences, $\frac{1}{\rho}$ is the elasticity of intertemporal substitution and $\delta$ is a subjective discount rate. The parameter $\zeta$ does not alter preferences, but gives some additional flexibility, and we will select it in a judicious manner. The stochastic discount factor $S$ for the recursive utility model satisfies:

$$
\frac{S_{t+1}}{S_{t}}=\exp (-\delta)\left(\frac{C_{t+1}}{C_{t}}\right)^{-\gamma}\left[\frac{V_{t+1} / C_{t+1}}{\mathcal{R}_{t}\left(V_{t+1} / C_{t}\right)}\right]^{\rho-\gamma} .
$$

The presence of the next-period continuation value in the one-period stochastic discount factor introduces a forward-looking component to valuation. It gives a channel by which investor beliefs matter. I now explore the consequences of making the forward-looking contribution to the one-period stochastic discount factor as potent as possible in a way that can be formalized mathematically. This relevant for the empirical literature as that literature is often led to select parameter configurations that feature the role of continuation values.

Following Hansen (2012) and Hansen and Scheinkman (2012b), we consider the following equation:

$$
E\left[\left(\frac{C_{t+1}}{C_{t}}\right)^{1-\gamma} \hat{e}\left(X_{t+1}\right) \mid X_{t}=x\right]=\exp (\hat{\eta}) \hat{e}(x)
$$


Notice that this eigenvalue equation has the same structure as (4) with $\left(C_{t}\right)^{1-\gamma}$ taking the place of $S_{t}$. The formula for the stochastic discount factor remains well defined in the limiting case as we let $(\zeta)^{1-\rho}$ tend to zero and $\delta$ decreases to ${ }^{6}$

$$
\frac{1-\rho}{1-\gamma-1} \hat{\eta}
$$

Then

$$
\frac{V_{t}}{C_{t}} \approx\left[\hat{e}\left(X_{t}\right)\right]^{1-\gamma}
$$

and

$$
S_{t} \approx \exp (-\hat{\eta} t)\left(\frac{C_{t}}{C_{0}}\right)^{-\gamma}\left[\frac{\hat{e}\left(X_{t}\right)}{\hat{e}\left(X_{0}\right)}\right]^{\frac{\rho-\gamma}{1-\gamma}} .
$$

Therefore, in the limiting case

$$
h(x)=\hat{e}(x)^{\frac{\rho-\gamma}{1-\gamma}}
$$

in (8).

\subsubsection{Altering martingale components}

Some distorted belief models of asset pricing feature changes that alter the martingale components. As I have already discussed, positive martingales with unit expectations imply changes in the probability distribution. They act as so-called Radon-Nikodym derivatives for changes that are absolutely continuous over any finite time interval. Suppose that $N$ is a martingale for which $\log N$ is an additive functional. Thus

$$
E\left(\frac{N_{t+1}}{N_{t}} \mid X_{t}=x\right)=1
$$

This martingale captures investors beliefs that can be distinct from those given by the underlying model specification. Since Assumption 2.1 is satisfied, for the baseline specification, it may be shown that the alternative probability specification induced by the martingale $N$ also satisfies the assumption. This hypothesized difference between the model and the beliefs of investors is presumed to be permanent with this specification. That is, investors have confidence in this alternative model and do not, for instance consider a mixture specification while attempting to infer the relative weights using historical data.

\footnotetext{
${ }^{6}$ Hansen and Scheinkman (2012b) use the associated change of measure to show when existence to the Perron-Frobenius problem implies the existence of a solution to the fixed point equation associated with an infinite-horizon investor provided that $\delta$ is less than this limiting threshold.
} 
For some distorted belief models, the baseline stochastic discount factor $S^{*}$ from power utility is altered by the martingale used to model the belief distortion:

$$
S=S^{*} N
$$

Asset valuation inherits the distortion in the beliefs of the investors. Consider factorization (7) for $S^{*}$. Typically $N M^{*}$ will not be a martingale even though both components are martingales. Thus to obtain the counterpart factorization for a distorted belief economy with stochastic discount factor $S$ requires that we extract a the martingale component from $N M^{*}$. Belief changes of this type have permanent consequences for asset valuation.

Examples of models with exogenous belief distortions that can be modeled in this way include Cecchetti et al. (2000) and Abel (2002). Related research by Hansen et al. (1999), Chen and Epstein (2002), Anderson et al. (2003) and Ilut and Schneider (2012) uses a preference for robustness to model misspecification and ambiguity aversion to motivate explicitly this pessimism. ${ }^{7}$ In this literature the form of the pessimism is an endogenous response to investors' uncertainty about which among a class of model probability specifications governs the dynamic evolution of the underlying state variables. The martingale $N$ is not their "actual belief" rather the outcome of exploring the utility consequences of considering an array of probability models. Typically there is a benchmark model that is used, and we take the model that we have specified without distortion as this benchmark. In these specifications, the model uncertainty does not vanish over time via learning because investors are perpetually reluctant to embrace a single probability model.

\subsubsection{Endogenous responses}

So far our discussion has held fixed the consumption process in order to simplify the impact of changing preferences. Some stochastic growth models with production have a balanced growth path relative to some stochastically growing technology. In such economies, some changes in preferences, while altering consumption allocations, may still preserve the martingale component along with the long-term interest rate.

\footnotetext{
${ }^{7}$ There is a formal link between some recursive utility specifications and robust utility specifications that has origins in the control theory literature on risk-sensitive control. Anderson et al. (2003) and Maenhout (2004) develop these links in models of portfolio choice and asset pricing.
} 


\subsection{Entropy characterization}

In the construction that follows we build on ideas from Bansal and Lehmann (1997), Alvarez and Jermann (2005), and especially Backus et al. (2011). The relative entropy of a stochastic discount factor functional $S$ for horizon $t$ is given by:

$$
\frac{1}{t}\left[\log E\left(S_{t} \mid X_{0}=x\right)-E\left(\log S_{t} \mid X_{0}=x\right)\right],
$$

which is nonnegative as an implication of Jensen's Inequality. When $S_{t}$ is log-normal, this notion of entropy yields one-half the conditional variance of $\log S_{t}$ conditioned on date zero information, and Alvarez and Jermann (2005) propose using this measure as a "generalized notion of variation." Backus et al. (2011) study this measure of relative entropy averaged over the initial state $X_{0}$. They view this entropy measure for different investment horizons as an attractive alternative to the volatility of stochastic discount factors featured by Hansen and Jagannathan (1991). To relate these entropy measures to asset pricing models and data, Backus et al. (2011) note that

$$
-\frac{1}{t} E\left[\log E\left(S_{t} \mid X_{0}\right)\right]
$$

is the average yield on a $t$-period discount bond where we use the stationary distribution for $X_{0}$. Following Bansal and Lehmann (1997),

$$
-\frac{1}{t} E\left(\log S_{t}\right)=-E\left(\log S_{1}\right)
$$

is the average one-period return on the maximal growth portfolio under the same distribution.

Borovicka and Hansen (2012) derive a more refined quantification of how entropy depends on the investment horizon $t$ given by

$$
\frac{1}{t}\left[\log E\left(S_{t} \mid X_{0}\right)-E\left(\log S_{t} \mid X_{0}\right)\right]=\frac{1}{t} \sum_{j=1}^{t} E\left[\varsigma\left(X_{t-j}, j\right) \mid X_{0}\right] .
$$

The right-hand side represents the horizon $t$ entropy in terms of averages of the building blocks $\varsigma(x, t)$ where

$$
\varsigma(x, t)=\log E\left[S_{t} \mid X_{0}=x\right]-E\left[\log E\left(S_{t} \mid \mathcal{F}_{1}\right) \mid X_{0}=x\right] \geq 0 .
$$


The term $\varsigma$ is itself a measure of "entropy" of

$$
\frac{E\left(S_{t} \mid \mathcal{F}_{1}\right)}{E\left(S_{t} \mid \mathcal{F}_{0}\right)}
$$

conditioned on date zero information and measures the magnitude of new information that arrives between date zero and date one for $S_{t}$. For log-normal models, $\varsigma(x, t)$ is one half the variance of $E\left(\log S_{t} \mid \mathcal{F}_{1}\right)-E\left(\log S_{t} \mid \mathcal{F}_{0}\right)$. 


\section{Cash-flow pricing}

Rubinstein (1976) pushed us to think of the asset pricing implications from a multi-period perspective in which an underlying set of future cash flows are priced. I adopt that vantage point here. Asset values can move either because market-determined stochastic discount rates have altered (a price change), or because the underlying claim implies a higher or lower cash flow (a quantity change). These two channels motivate formal methods for enhancing our understanding of what economic models have to say about present-value relations. One common approach uses log-linear approximation to identify two (correlated) sources of time variation in the ratio of an asset value to the current period cash flow. The first source is time variation in expected returns to holding the asset, a price effect; and the second is time variation in expected dividend growth rates, a quantity effect. Here I explore some more broadly applicable methods to produce "dynamic valuation decompositions" which are complementary to the log-linear approach. My aim is to unbundle the pricing of cash flows in revealing ways. The specific impetus for this formulation comes form the work of Lettau and Wachter (2007) and Hansen et al. (2008), and the general formulation follows Hansen and Scheinkman (2009) and Hansen (2012).

\subsection{Incorporating stochastic growth in the cash flows}

Let $G$ be a stochastic growth factor where $\log G$ satisfies Assumption 2.1. Notice that if $\log G$ and $\log S$ both satisfy this assumption, their sum does as well. While the stochastic discount factor decays over time, the stochastic growth factor grows over time. I will presume that discounting dominates and that the product $S G$ is expected to decay over time. I consider cash flows of the type:

$$
G_{t+1} \phi\left(Y_{t+1}-Y_{t}, X_{t+1}\right)
$$

where $G_{0}$ is in the date zero information set $\mathcal{F}$. The date $t$ value of this cash flow is:

$$
E\left[\frac{S_{t+1}}{S_{0}} G_{t+1} \phi\left(Y_{t+1}-Y_{t}, X_{t+1}\right) \mid \mathcal{F}_{0}\right]=G_{0} E\left[\frac{S_{t+1} G_{t+1}}{S_{0} G_{0}} \phi\left(Y_{t+1}-Y_{t}, X_{t+1}\right) \mid X_{0}\right] .
$$


An equity sums the values of the cash flows at all dates $t=1,2, \ldots$. By design we may compute values recursively repeatedly applying a one-period valuation operator:

$$
\mathbb{V} h(x)=E\left[\frac{S_{t+1} G_{t+1}}{S_{t} G_{t}} h\left(x_{t+1}\right) \mid X_{t}=x\right] .
$$

Let

$$
h(x)=E\left[\frac{S_{t+1} G_{t+1}}{S_{t} G_{t}} \phi\left(Y_{t+1}-Y_{t}, X_{t+1}\right) \mid X_{t}=x\right]
$$

Then

$$
E\left[\frac{S_{t+1}}{S_{0}} G_{t+1} \phi\left(Y_{t+1}-Y_{t}, X_{t+1}\right) \mid \mathcal{F}_{0}\right]=G_{0} \mathbb{V}^{t} h(x) .
$$

To study cash flow pricing with stochastic growth factors, we use a factorization of the type given in (3) but applied to $S G$ instead of $S$ :

$$
\frac{S_{t} G_{t}}{S_{0} G_{0}}=\exp (-\eta t)\left(\frac{M_{t}}{M_{0}}\right)\left[\frac{f\left(X_{t}\right)}{f\left(X_{0}\right)}\right]
$$

where $f=\frac{1}{e}$ and $e$ solves:

$$
E\left[\left(\frac{S_{t+1} G_{t+1}}{S_{t} G_{t}}\right) e\left(X_{t+1} \mid X_{t}=x\right]=\exp (-\eta) e(x) .\right.
$$

The factorization of $S G$ cannot be obtained by factoring $S$ and $G$ separately and multiplying the outcome because products of martingales are not typically martingales. Thus codependence matters. ${ }^{8}$

\subsection{Holding-period returns on cash flows}

A return to equity with cash flows or dividends that have stochastic growth components can be viewed as a bundle or portfolios of holding period returns on cash flows with alternative payout dates. (See Lettau and Wachter (2007) and Hansen et al. (2008).) The gross one-period holding-period return over a payoff horizon $t$ is:

$$
\left(\frac{G_{1}}{G_{0}}\right)\left(\frac{\mathbb{V}_{t-1}\left[h\left(X_{1}\right)\right]}{\mathbb{V}_{t}\left[h\left(X_{0}\right)\right]}\right)
$$

\footnotetext{
${ }^{8}$ When $S$ and $G$ are jointly lognormally distributed, we may first extract martingale components of log $S$ and $\log G$ and add these together and exponentiate. While this exponential will not itself be a martingale, we may construct a positive martingale by multiplying this exponential by a geometrically declining scale factor.
} 
Changing the payoff date $t$ changes the exposure through a valuation channel as reflected by the second term in brackets, while the direct cash flow channel reflected by the first term remains the same as we change the payoff horizon.

To characterize the holding-period return for large $t$, I apply the change in measure and represent this return as:

$$
\exp (\eta) \frac{G_{1}}{G_{0}}\left[\frac{e\left(X_{1}\right)}{e\left(X_{0}\right)}\right]\left(\frac{\widetilde{E}\left[h\left(X_{t}\right) f\left(X_{t}\right) \mid X_{1}\right]}{\widetilde{E}\left[h\left(X_{t}\right) f\left(X_{t}\right) \mid X_{0}\right]}\right)
$$

The last term converges to unity as the payoff horizon $\tau$ increases, and the first two terms do not depend on $\tau$. Thus the limiting return is:

$$
\left(\frac{G_{1}}{G_{0}}\right)\left[\exp (\eta) \frac{e\left(X_{1}\right)}{e\left(X_{0}\right)}\right]
$$

The valuation component is now tied directly to the solution to the Perron-Frobenius problem. An eigenfunction ratio captures the state dependence. In addition there is an exponential adjustment $\eta$, which is in effect a value-based measure of duration of the cash flow $G$ and is independent of the Markov state. When $\eta$ is near zero, the cash flow values deteriorate very slowly as the investment horizon is increased.

The study of holding-period returns on cash flows payoffs over alternative payoff dates gives one way to characterize a valuation dynamics. Recent work by van Binsbergen et al. (2011) and van Binsbergen et al. (2012) develop and explore empirical counterpart to these returns. Next I appeal to ideas from price theory to give a different depiction.

\subsection{Shock elasticities}

Next I develop valuation counterparts to impulse-response functions commonly used in the study of dynamic, stochastic equilibrium models. I refer to these counterparts as shock elasticities. As I will show, these elasticities measure both exposure and price sensitivity over alternative investment horizons.

As a starting point, consider a cash flow $G$ and stochastic discount factor $S$. For investment horizon $t$, form the logarithm of the expected return to this cash flow given by:

$$
\log E\left[\left(\frac{G_{t}}{G_{0}}\right) \mid X_{0}=x\right]-\log E\left[\left(\frac{G_{t}}{G_{0}}\right)\left(\frac{S_{t}}{S_{0}}\right) \mid X_{0}=x\right]
$$


where the scaling by $G_{0}$ is done for convenience. The first term is the logarithm of the expected payoff and the second term is the logarithm of the price. To measure the riskpremium I compare this expected return to a riskless investment over the same time horizon. This is a special case of my previous calculation in which I set $G_{t}=1$ for all $t$. Thus the logarithm of this returns is:

$$
-\log E\left[\left(\frac{S_{t}}{S_{0}}\right) \mid X_{0}=x\right]
$$

I measure the risk premium by comparing these two investments:

$$
\begin{aligned}
\text { risk premium }= & \log E\left[\left(\frac{G_{t}}{G_{0}}\right) \mid X_{0}=x\right]-\log E\left[\left(\frac{G_{t}}{G_{0}}\right)\left(\frac{S_{t}}{S_{0}}\right) \mid X_{0}=x\right] \\
& +\log E\left[\left(\frac{S_{t}}{S_{0}}\right) \mid X_{0}=x\right] .
\end{aligned}
$$

In what follows I will study the value implications as measured by what happens to the risk premium when I perturb the exposure of the cash flow to the underlying shocks.

To unbundle value implications, I borrow from price theory by computing shock price and shock exposure elasticities. (I think of an exposure elasticity as the counterpart to a quantity elasticity.) In so doing I build on the continuous-time analyses of Hansen and Scheinkman (2012a) and Borovička et al. (2011) and on the discrete-time analysis of Borovicka and Hansen (2012). To simplify the interpretation, suppose there is an underlying sequence of iid multivariate standard normally distributed shocks $\left\{W_{t+1}\right\}$. Introduce:

$$
\log H_{t+1}(\mathrm{r})-\log H_{t}(\mathrm{r})=\mathrm{r} \sigma\left(X_{t}\right) \cdot W_{t+1}-\frac{(\mathrm{r})^{2}}{2}\left|\sigma\left(X_{t}\right)\right|^{2}
$$

where I assume that

$$
E\left[\left|\sigma\left(X_{t}\right)\right|^{2}\right]=1
$$

and $\log H_{0}(\mathbf{r})=0$. Here I use $\sigma(x)$ to select the combination of shocks that is of interest and I scale this state-dependent vector in order that $\sigma\left(X_{t}\right) \cdot W_{t+1}$ has a unit standard deviation. ${ }^{9}$

Also I have constructed the increment in $\log H_{t+1}$ so that

$$
E\left[\frac{H_{t+1}(\mathrm{r})}{H_{t}(\mathrm{r})} \mid X_{t}=x\right]=1 .
$$

\footnotetext{
${ }^{9}$ Borovička et al. (2011) suggest counterpart elasticities for discrete states modeled as Markov processes.
} 
I use the resulting process $H(\mathrm{r})$ to define a scalar family of martingale perturbations parameterized by $r$.

Consider a cash flow $G$ that may grow stochastically over time. By multiplying $G$ by $H(\mathrm{r})$, I alter the exposure of the cash flow to shocks. Since I am featuring small changes, I am led to use the process:

$$
D_{t+1}-D_{t}=\sigma\left(X_{t}\right) \cdot W_{t+1}
$$

with $D_{0}=0$ to represent two exposure elasticities:

$$
\begin{array}{r}
\epsilon_{e}(x, t)=\left.\frac{d}{d \mathbf{r}} \frac{1}{t} \log E\left[\frac{G_{t}}{G_{0}} H_{t}(\mathrm{r}) \mid X_{0}=x\right]\right|_{\mathrm{r}=0}=\frac{1}{t} \frac{E\left[\frac{G_{t}}{G_{0}} D_{t} \mid X_{0}=x\right]}{E\left[\frac{G t}{G_{0}} \mid X_{0}=x\right]} \\
\varepsilon_{e}(x, t)=\left.\frac{d}{d \mathbf{r}} \log E\left[\frac{G_{t}}{G_{0}} H_{1}(\mathrm{r}) \mid X_{0}=x\right]\right|_{\mathrm{r}=0}=\sigma\left(X_{0}\right) \frac{E\left[\frac{G_{t}}{G_{0}} W_{1} \mid X_{0}=x\right]}{E\left[\frac{G t}{G_{0}} \mid X_{0}=x\right]} .
\end{array}
$$

These elasticities depend both on the investment horizon $t$ and the current value of the Markov state $x$. For a fixed horizon $t$, the first of these elasticities, which I call a risk-price elasticity, changes the exposure at all horizons. The second one concentrates on changing only the first period exposure, much like an impulse response function. ${ }^{10}$ As argued by Borovička et al. (2011) and Borovicka and Hansen (2012), the risk price-elasticities are weighted averages of the shock-price elasticities.

The long-term limit (as $t \rightarrow \infty$ ) of the shock-price elasticity has a tractable characterization. Consider a factorization of the form (3), but applied to $G$. Using the martingale from this factorization, Borovicka and Hansen (2012) show that

$$
\lim _{t \rightarrow \infty} \frac{E\left[\frac{G_{t}}{G_{0}} W_{1} \mid X_{0}=x\right]}{E\left[\frac{G_{t}}{G_{0}} \mid X_{0}=x\right]}=\widetilde{E}\left[W_{1} \mid X_{0}=x\right] .
$$

\footnotetext{
${ }^{10}$ Under log-normality there is a formal equivalence between our elasticity and an impulse response function.
} 
As intermediate calculations, I also compute:

$$
\begin{aligned}
& \epsilon_{v}(x, t)=\left.\frac{d}{d r} \frac{1}{t} \log E\left[\frac{S_{t} G_{t}}{S_{0} G_{0}} H_{t}(\mathbf{r}) \mid X_{0}=x\right]\right|_{\mathrm{r}=0}=\frac{1}{t} \frac{E\left[\frac{S_{t} G_{t}}{S_{0} G_{0}} D_{t} \mid X_{0}\right]}{E\left[\frac{S_{t} G_{t}}{S_{0} G_{0}} \mid X_{0}\right]} \\
& \varepsilon_{v}(x, t)=\left.\frac{d}{d \mathbf{r}} \log E\left[\frac{S_{t} G_{t}}{S_{0} G_{0}} H_{1}(\mathrm{r}) \mid X_{0}=x\right]\right|_{\mathrm{r}=0}=\frac{E\left[\frac{S_{t} G_{t}}{S_{0} G_{0}} D_{1} \mid X_{0}\right]}{E\left[\frac{S_{t} G_{t}}{S_{0} G_{0}} \mid X_{0}\right]}
\end{aligned}
$$

which measure the sensitivity of value to changes in the exposure. These elasticities incorporate both a change in price and a change in exposure. The implied risk-price and shock-price elasticities are given by:

$$
\begin{aligned}
& \epsilon_{p}(x, t)=\epsilon_{e}(x, t)-\epsilon_{v}(x, t) \\
& \varepsilon_{p}(x, t)=\varepsilon_{e}(x, t)-\varepsilon_{v}(x, t) .
\end{aligned}
$$

In what follows I draw on some illustrations from the existing literature.

\subsubsection{Lettau-Wachter example}

Lettau and Wachter (2007) consider an asset pricing model of cash-flow duration. They use an ad hoc model of a stochastic discount factor to display some interesting patterns of risk premia. When thinking about the term structure of risk premia, I find it useful to distinguish pricing implications from exposure implications. Both can contribute to risk premia as a function of the investment horizon.

Lettau and Wachter (2007) explore implications of a cash flow process with linear dynamics:

$$
X_{t+1}=\left[\begin{array}{cc}
.9658 & 0 \\
0 & .9767
\end{array}\right] X_{t}+\left[\begin{array}{ccc}
.12 & 0 & 0 \\
0 & -.0013 & .0009
\end{array}\right] W_{t+1}
$$

where $\left\{W_{t+1}\right\}$ is iid multivariate standard normally distributed. They model the logarithm of the cash flow process as

$$
\log G_{t+1}-\log G_{t}=\mu_{g}+X_{t}^{[2]}+\left[\begin{array}{lll}
0 & .0724 & 0
\end{array}\right] W_{t+1}
$$

where $X_{t}^{[2]}$ is the second component of $X_{t}$. I compute shock exposure elasticities, which in this case are essentially the same as impulse response functions for $\log G$ since the cash 
flow process is log-normal. The exposure elasticities for the two shocks are depicted in the top panel of Figure 1.
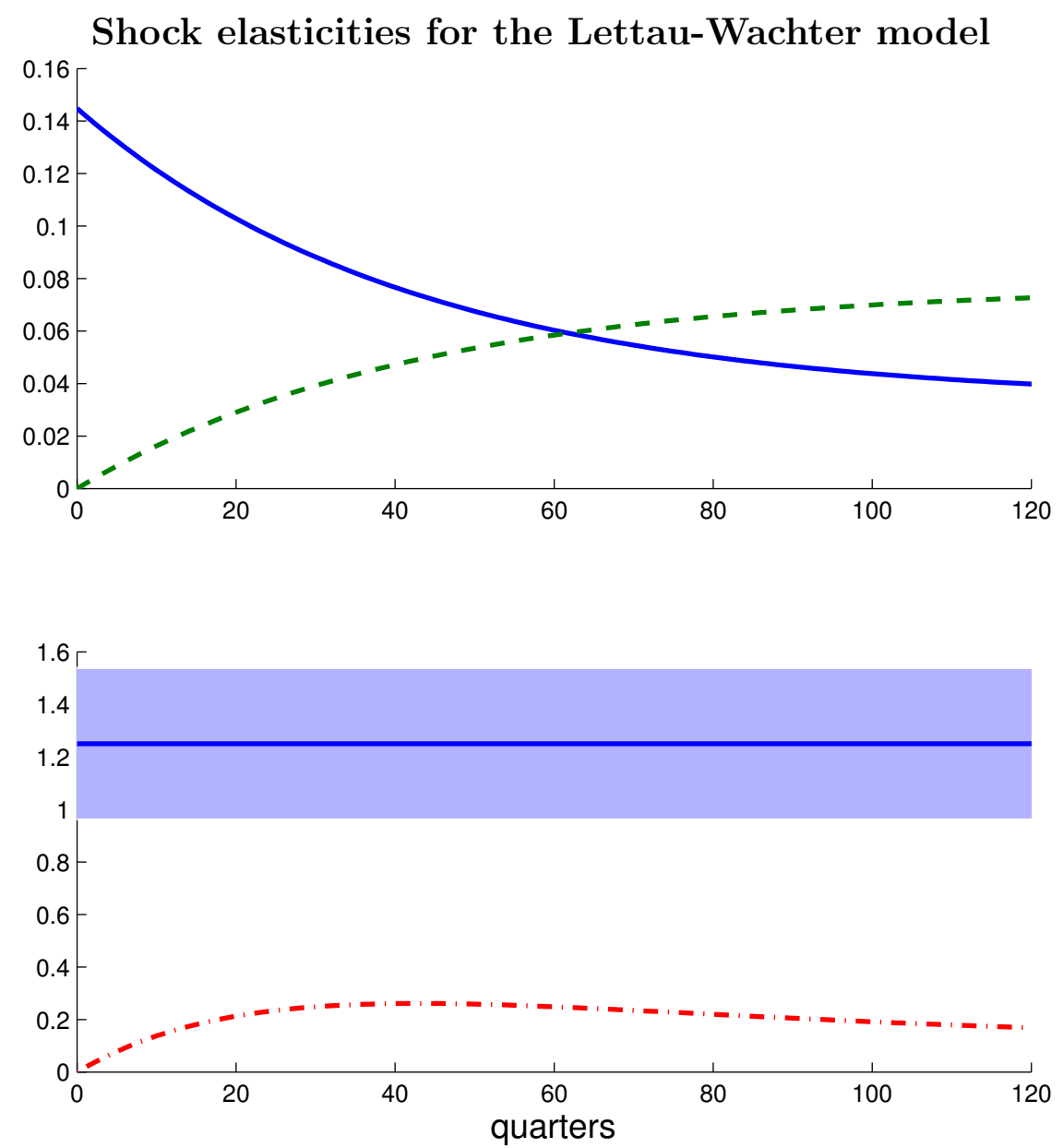

Figure 1: The top panel of this figure depicts the shock-exposure elasticities for the second (solid blue line) and third (dashed green line) shocks obtained by setting $\sigma$ to be the corresponding coordinate vectors. The shock-exposure elasticities for the first shock are zero. The bottom panel of this figure depicts the shock-price elasticities for the first shock (dotted red line) and for the second shock (solid blue line) over alternative investment horizons. The shock-price elasticities for the third shock are zero. The shaded area gives the interquartile range for the shock price elasticities implied by state dependence.

For shock two, the immediate exposure dominates that long-run response. In contrast the third shock exposure starts at zero builds to a positive limit, but at a value that is notably higher than the second shock. 
Next we assign "prices" to the shock exposures. The stochastic discount factor in Lettau-Wachter model evolves as:

$$
\log S_{t+1}-\log S_{t}=-r-\left(.625+X_{t}^{[1]}\right)\left[\begin{array}{lll}
0 & 1 & 0
\end{array}\right] W_{t+1}-\frac{\left|.625+X_{t}^{[1]}\right|^{2}}{2}
$$

Nonlinearity is present in this model because the conditional mean of $\log S_{t+1}-\log S_{t}$ is quadratic in $X_{t}^{[1]}$. This is a model with a constant interest rate $r$ and state dependent one-period shock price vector:

$$
\left(.625+X_{t}^{[1]}\right)\left[\begin{array}{l}
0 \\
1 \\
0
\end{array}\right]
$$

By assumption only the second shock commands a nonzero one-period shock price elasticity and this elasticity varies over time. The process $\left\{.625+X_{t}^{[1]}\right\}$ is a stochastic volatility process that induces movements in the shock price elasticities. In its stationary distribution, this process has a standard deviation of .46 and hence varies substantially relative to its mean of .625. The first shock alters the first component of $X_{t}$ and the shock-price elasticity for the first shock is different from zero after one period. The cash flow $G$ does not respond to this shock so the "pricing" of the first component of $W_{t+1}^{[1]}$ does not play a direct role in the valuation of $G{ }^{11}$

The shock-price elasticities are depicted in the bottom panel of Figure 1. A consequence of the specification of the stochastic discount factor $S$ is that the second shock has a constant (but state dependent) shock-price elasticity of $.625+X_{t}^{[1]}$ as a function of the investment horizon. This shock has the biggest impact for the cash flow, and it commands the largest shock price elasticity elasticity both immediately and over the long term. Thus, I have shown that this application of dynamic value decomposition reveals that the impetus for the downward risk premia as a function of horizon comes from the dynamics of the cash-flow shock exposure and not from the price elasticity of that exposure.

We now shift to a different specification of preferences and cash flows, and show what this same methods reveal in a different context.

\footnotetext{
${ }^{11}$ Lettau and Wachter (2007) use this model to interpret the differential expected returns in growth and value stocks. Value stocks are more exposed to the second shock .
} 


\subsubsection{Recursive utility}

We illustrate pricing implications for the recursive utility model using a specification from Hansen et al. (2007) of a "long-run risk" model for consumption dynamics featured by Bansal and Yaron (2004). Bansal and Yaron (2004) use historical data from the United States to motivate their model including the choice of parameters. Their model includes predictability in both conditional means and in conditional volatility. We use the continuoustime specification from Hansen et al. (2007) because the continuous-time specification of stochastic volatility is more tractable:

$$
\begin{aligned}
d X_{t}^{[1]} & =-.021 X_{t}^{[1]} d t+\sqrt{X_{t}^{[2]}}\left[\begin{array}{lll}
.00031 & -.00015 & 0
\end{array}\right] d W_{t}, \\
d X_{t}^{[2]} & =-.013\left(X_{t}^{[2]}-1\right) d t+\sqrt{X_{t}^{[2]}}\left[\begin{array}{lll}
0 & 0 & -.038
\end{array}\right] d W_{t} \\
d \log C_{t} & =.0015 d t+X_{t}^{[1]} d t+\sqrt{X_{t}^{[2]}}\left[\begin{array}{lll}
.0034 & 0.007 & 0
\end{array}\right] d W_{t},
\end{aligned}
$$

where $W$ is a trivariate standard Brownian motion. The unit of time in this time series specification is one month, although for comparability with other models I plot shock-price elasticities using quarters as the unit of time. The first component of the state vector is the state dependent component to the conditional growth rate, and the second component is a volatility state. Both the growth state and the volatility state are persistent. We follow Hansen (2012) in configuring the shocks for this example. The first one is the "permanent shock" identified using standard time series methods and normalized to have a unit standard deviation. The second shock is a so-called temporary shock, which by construction is uncorrelated with the first shock.

Our analysis assumes a discrete-time model. A continuous-time Markov process $X$ observed at say interval points in time remains a Markov process in discrete time. Since $\log C_{t+1}-\log C_{t}$ is constructed via integration, it is not an exact function of $X_{t+1}$ and $X_{t}$. To apply our analysis, we define $Y_{t+1}=\log C_{t+1}-\log C_{t}$. Given the continuous-time Markov specification, the joint distribution of $\log C_{t+1}-\log C_{t}$ and $X_{t+1}$ conditioned on past information only depends on the current Markov state $X_{t}$ as required by Assumption 2.1. ${ }^{12}$ The resulting shock-price elasticities are reported in Figure 2 for the three different shocks. Since the model with power utility $(\rho=\gamma=8)$ has preferences that are additively separable, the pricing impact of a permanent shock or a stochastic-volatility shock accu-

\footnotetext{
${ }^{12}$ I exploit the continuous-time quasi analytical formulas given by Hansen (2012) for the actual computations.
} 
mulates over time with the largest shock-price elasticities at the large investment horizon limit. In contrast, recursive utility with $(\rho=1, \gamma=8)$ has an important forward-looking component for pricing. ${ }^{13}$ As a consequence, the trajectory for the shock-price elasticities for the permanent shock and for the shock to stochastic volatility are much flatter than for the power utility model, and in particular, the short-term shock price elasticity is relatively large for the permanent shock to consumption.

The presence of stochastic volatility induces state dependence in all of the the shockprice elasticities. This dependence is reflected in the shaded portions in Figure 2 and of particular interest for the permanent shock, and its presence is a source of time variation in the elasticities for each of the investment horizons.

The amplification of the short-term shock price elasticities has been emphasized at the outset in the literature on "long run risk" through the guises of the recursive utility model. Figure 2 provides a more complete picture of cash risk pricing. The fact the limiting behavior for recursive and power utility specifications are in agreement follow from the factorization (11).

Models with external habit persistence provide a rather different characterization of shock price elasticities as I will now illustrate.

\subsubsection{External habit models}

Borovička et al. (2011) provide a detailed comparison of the pricing implications of two specifications of external habit persistence, one given in Campbell and Cochrane (1999) and the other in Santos and Veronesi (2006). In order to make the short-term elasticities comparable, Borovička et al. (2011) modified the parameters for the Santos and Veronesi (2006) model. Borovička et al. (2011) performed their calculations using a continuous-time specification in which consumption is a random walk with drift when specified in logarithms. Thus, in contrast to the "long-run risk model", the consumption exposure elasticities are constant.

$$
d \log C_{t}=.0054 d t+.0054 d W_{t}
$$

where $W$ is a scalar standard Brownian motion and the numerical value of $\mu_{c}$ is inconsequential to our calculations. I will not elaborate on the precise construction of the social habit stock used to model the consumption externality and instead posit the implied stochas-

\footnotetext{
${ }^{13}$ See Hansen (2012) for a discussion of the sensitivity to the parameter $\rho$, which governs the intertemporal elasticity of substitution.
} 


\section{Shock-price elasticities for recursive utility model}
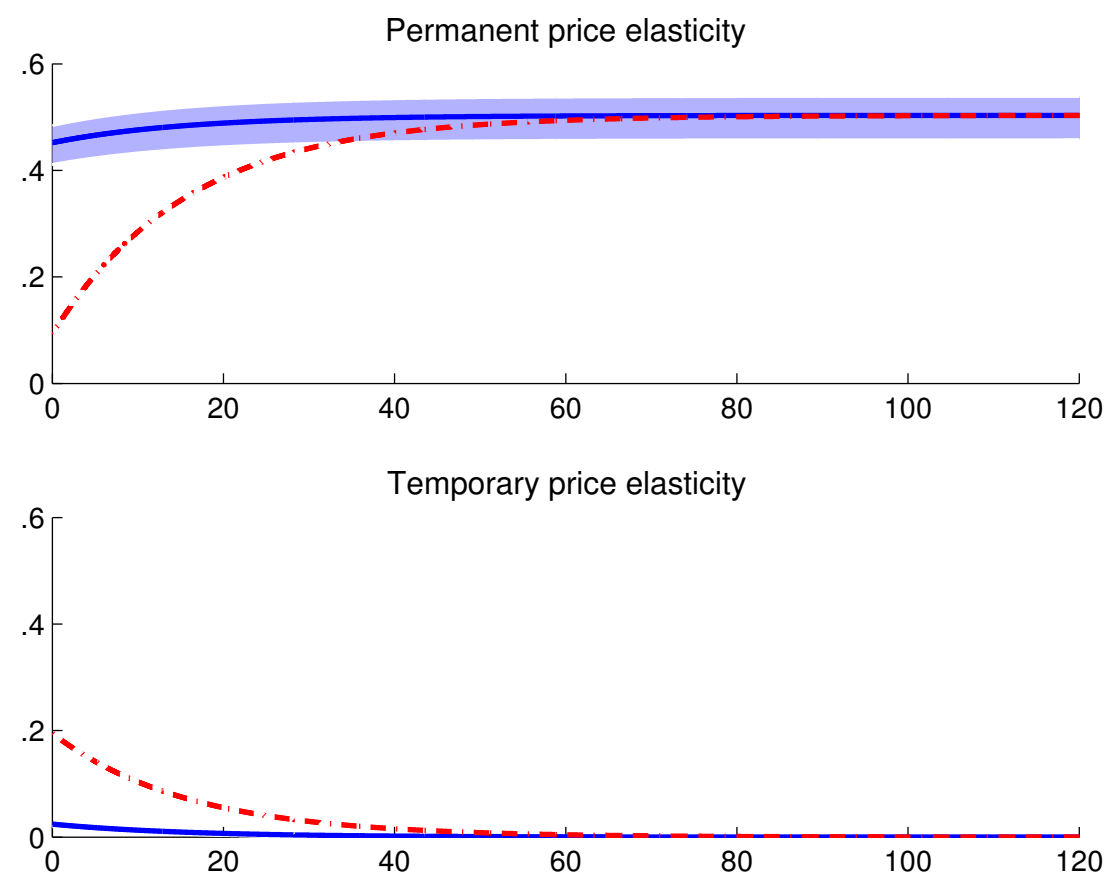

Volatility price elasticity

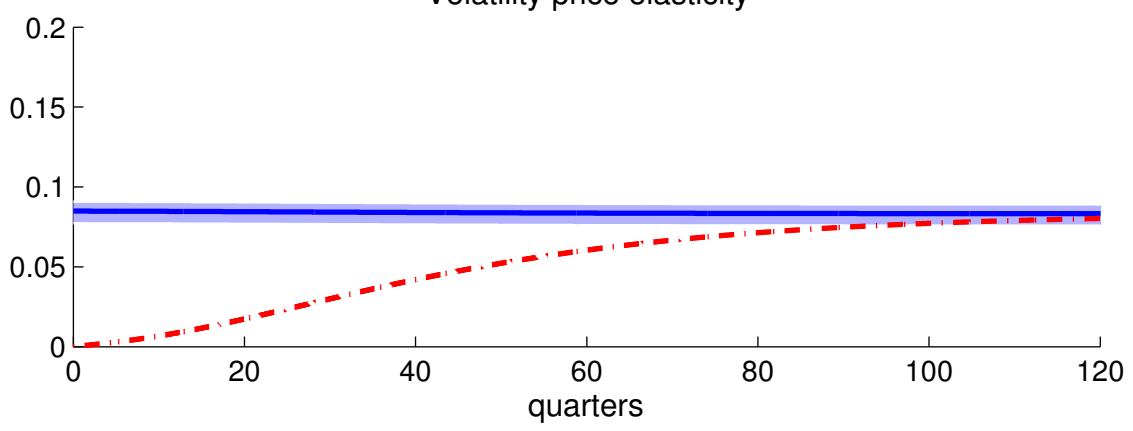

Figure 2: This figure depicts the shock-price elasticities of the three shocks for a model with power utility $(\rho=\gamma=8)$ depicted by the dashed red line and with recursive utility $(\rho=1, \gamma=8)$ depicted by the solid blue line. The shaded region gives the interquartile range of the shock price elasticities induced by state dependence for the recursive utility model.

tic discount factors. The constructions differ and are delineated in the respective papers. Rather than embrace a full structural interpretation of the consumption externality, I will focus on the specification of the stochastic discount factors for the two models. 
For Santos and Veronesi (2006), the stochastic discount factor is

$$
\frac{S_{t}}{S_{0}}=\exp (-\delta t)\left(\frac{C_{t}}{C_{0}}\right)^{-2} \frac{X_{t}+1}{X_{0}+1}
$$

where

$$
d X_{t}=-.035\left(X_{t}-2.335\right) d t-.496 d W_{t} .
$$

Thus the shock to $d X_{t}$ is proportional to the shock to $d \log C_{t}$ with the same magnitude but opposite sign. In our calculations we set $G=C$. Consequently, the martingale component to the stochastic discount factor is given by

$$
\frac{M_{t}}{M_{0}}=\exp \left[(.0054) W_{t}-W_{0}-\frac{t}{2}(.0054)^{2}\right]
$$

and the Perron-Frobenius eigenfunction is $e(x)=\frac{1}{x+1}$.

For Campbell and Cochrane (1999), the stochastic discount factor is

$$
\frac{S_{t}}{S_{0}}=\exp (-\delta t)\left(\frac{C_{t}}{C_{0}}\right)^{-2} \frac{\exp \left(2 X_{t}\right)}{\exp \left(2 X_{0}\right)}
$$

where

$$
d X_{t}=-.035\left(X_{t}-.4992\right)+\left(1-\sqrt{1+1200 X_{t}}\right) d W_{t} .
$$

In this case the Perron-Frobenius eigenfunction is $e(x)=\exp (-2 x)$. The martingale components of $S$ are the same for the two models, as are the martingale components for $S G$.

Figure (3) depicts the shock-price elasticities for the two models for the quartiles of the state distribution. While the starting points and limit points for the shock-price trajectories agree, there is a substantial difference how fast the trajectories approach their limits. The long-term limit point is the same as that for a power utility specification $(\rho=\gamma=2)$. For the Santos and Veronesi (2006) specification, the consumption externality is arguably a transient model component. For the Campbell and Cochrane (1999) specification, this externality has very durable pricing implications even if formally speaking this model feature is transient. The nonlinearities in the state dynamics apparently compound in a rather different manner for the two specifications. See Borovička et al. (2011) for a more extensive comparison and discussion.

These examples all feature models with directly specified consumption dynamics. While this has some pedagogical simplicity for comparing impact of investor preferences on asset 


\section{Shock-price elasticities for the external habit model}
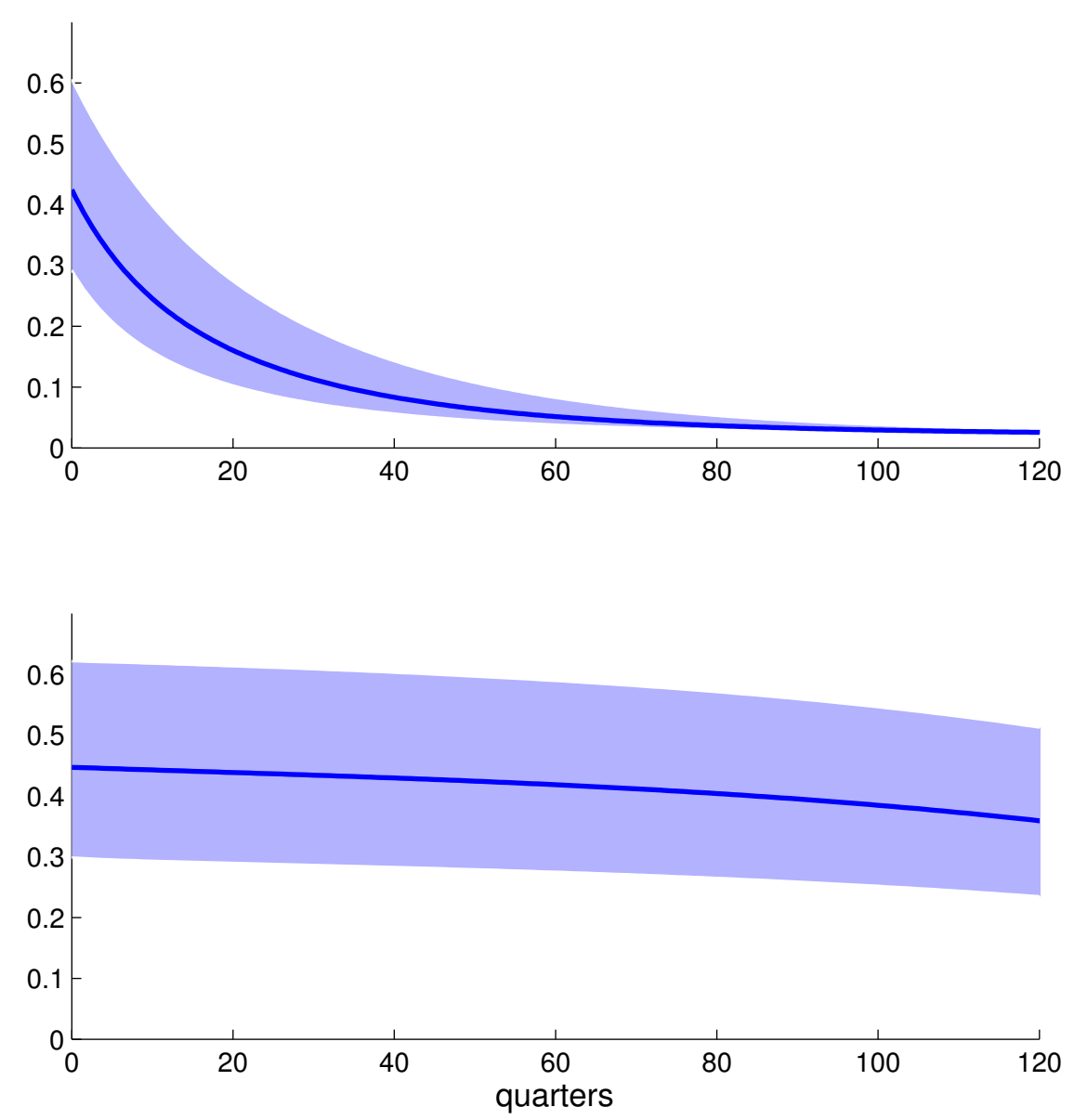

Figure 3: This figure depicts the shock-price elasticities for this single shock specification of the models with consumption externalities. The top panel displays the shock-price elasticity function in the Santos and Veronesi (2006) specification, while the bottom panel displays the Campbell and Cochrane (1999) specification. The solid curve conditions on the median state, while the shaded region depicts the interquartile range induced by state dependence.

prices, it is of considerable interest to apply these dynamic value decomposition (DVD) methods to a richer class of economies including economies with multiple capital stocks. For example, Borovicka and Hansen (2012) apply the methods to study a production economy with "tangible" and "intangible" capital as modeled in Ai et al. (2010). Richer models will provide scope for analyzing the impact of shock exposures with more interesting economic interpretations. 
The elasticities displayed here are local in nature. They feature small changes in exposure to normally distributed shocks. For highly nonlinear models, global alternatives may well have some appeal; or at the very least alternative ways to alter exposure to non-gaussian tail risk. 


\section{Market Restrictions}

I now explore the stochastic discount factors that emerge from some benchmark economies in which there is imperfect risk sharing. In part, my aim is to provide a characterization about how these economies relate to the more commonly used structural models of asset pricing. The cross-sectional distribution of consumption matters in these examples, and this presents interesting challenges for empirical implementation. While acknowledging these challenges, my goal is to understand how these distributional impacts are encoded in asset prices over alternative investment horizons.

I study some alternative benchmark economies with equilibrium stochastic discount factor increments that can be expressed as:

$$
\frac{S_{t+1}}{S_{t}}=\left(\frac{S_{t+1}^{a}}{S_{t}^{a}}\right)\left(\frac{S_{t+1}^{c}}{S_{t}^{c}}\right)
$$

where the first-term on the right-hand side, $\frac{S_{t+1}^{a}}{S_{t}^{a}}$, coincides with that of a representative consumer economy and the second term, $\frac{S_{t+1}^{c}}{S_{t}^{c}}$, depends on the cross-sectional distribution of consumption relative an average or aggregate. In the examples that I explore,

$$
\frac{S_{t+1}^{a}}{S_{t}^{a}}=\exp (-\delta)\left(\frac{C_{t+1}^{a}}{C_{t}^{a}}\right)^{-\rho}
$$

where $C^{a}$ denotes aggregate consumption. The way in which $S^{c}$ depends on the cross section differs in the example economies that I discuss because the market restrictions differ. As in the literature that I discuss, I allow the cross-sectional distribution of consumption (relative to an average) to depend on aggregate states.

While a full characterization of the term structure implications for risk prices is a worthy goal, here I will only initiate such a discussion by investigating when these limits on risk sharing lead to "transient" vs. "permanent" implications for market values. In one case below, $S_{t}^{c}=f\left(X_{t}\right)$ for some (Borel measurable) function $f$ of a stochastically stable process $X$. Thus we know that introducing market imperfections has only transient consequences. For the other examples, I use this method to indicate what are the sources within the model for long-term influence of cross-sectional consumption distributions on asset values. 


\subsection{Incomplete contracting}

Our first two examples are economies in which there are aggregate, public shocks and idiosyncratic, private shocks. Payoffs can be written on the public shocks but not on the private shocks. Let $\mathcal{G}_{t}$ denote the sigma algebra that includes both public and private shocks, and let $\mathcal{F}_{t}$ denote the sigma algebra that includes only public shocks. By forming expectations of date $t$ random variables that are $\mathcal{G}_{t}$ measurable conditioned on $\mathcal{F}_{t}$, we aggregate over the idiosyncratic shocks but condition on the aggregate shocks. We use this device to form cross-sectional averages. I presume that that

$$
E\left(Q_{t+1} \mid \mathcal{G}_{t}\right)=E\left(Q_{t+1} \mid \mathcal{F}_{t}\right)
$$

whenever $Q_{t+1}$ is $\mathcal{F}_{t}$ measurable. There could be time invariant components to the specification of $\mathcal{G}_{t}$, components that reflect an individual's type.

In what follows I use $C_{t}$ to express consumption in a manner that implicitly includes dependence on idiosyncratic shocks. Thus $C_{t}$ is $\mathcal{G}_{t}$ measurable. Thus the notation $C_{t}$ includes a specification of consumption allocated to a cross section of individuals at date $t$. With this notation, aggregate consumption is:

$$
C_{t}^{a}=E\left(C_{t} \mid \mathcal{F}_{t}\right)
$$

As an example, following Constantinides and Duffie (1996) consider consumption allocations of the type:

$$
\log C_{t+1}-\log C_{t}=\log C_{t+1}^{a}-\log C_{t}^{a}+V_{t+1} Z_{t+1}-\frac{1}{2}\left(Z_{t+1}\right)^{2}
$$

where $V_{t+1}$ is $\mathcal{G}_{t+1}$ measurable and a standard normally distributed random variable conditioned on composite event collection: $\mathcal{G}_{t} \vee \mathcal{F}_{t+1}$. The random variable $Z_{t+1}$ is in the public information set $\mathcal{F}_{t+1}$. It now suffices to define the cross-sectional average

$$
C_{0}^{a}=E\left[C_{0} \mid \mathcal{F}_{0}\right]
$$

then (17) is satisfied because

$$
E\left(\exp \left[V_{t+1} Z_{t+1}-\frac{1}{2}\left(Z_{t+1}\right)^{2}\right] \mid \mathcal{G}_{t} \vee \mathcal{F}_{t+1}\right)=1 .
$$


In this example since $V_{t+1}$ is an idiosyncratic shock, the idiosyncratic contribution to aggregate consumption has permanent consequences the aggregate random variable $Z_{t+1}$ shifts the cross sectional consumption distribution. Shortly we will discuss a decentralization that accompanies this distribution for which aggregate uncertainty in the cross-sectional distribution of consumption matters for valuation. This is just an example, and more general and primitive starting points are of interest.

In what follows, to feature the role of market structure we assume a common discounted power utility function for consumers $\rho=\gamma$. The structure of the argument is very similar to that of Kocherlakota and Pistaferri (2009), but there are some differences. ${ }^{14}$ Of course one could "add on" a richer collection of models of investor preferences, and for explaining empirical evidence there may be good reason to do so. To exposit the role of market structure, I focus on a particularly simple specification of consumer preferences.

\subsubsection{Trading assets that depend only on aggregate shocks}

First I consider a decentralized economy in which heterogenous consumers trade securities with payoffs that only depend on the aggregate states. Markets are incomplete because consumers cannot trade

I introduce a random variable $Q_{t+1}$ that is $\mathcal{F}_{t+1}$ measurable. Imagine adding $\mathrm{r} Q_{t+1}$ to the $t+1$ consumption utilities. The date $t$ price of the payoff $\mathrm{r} Q_{t+1}$ is

$$
\mathrm{r} E\left[\left(\frac{S_{t+1}}{S_{t}}\right) Q_{t+1} \mid \mathcal{F}_{t}\right]
$$

which must be subtracted from the date $t$ consumption. The scalar $r$ can be $\mathcal{G}_{t}$ measurable. We consider an equilibrium allocation for $C$, and thus part of the equilibrium restriction is that $r=0$ be optimal. This leads to the first-order conditions:

$$
\left(C_{t}\right)^{-\rho} E\left[\left(\frac{S_{t+1}}{S_{t}}\right) Q_{t+1} \mid \mathcal{F}_{t}\right]=\exp (-\delta) E\left[\left(C_{t+1}\right)^{-\rho} Q_{t+1} \mid \mathcal{G}_{t}\right]
$$

In order to feature the cross sectional distribution of consumption, I construct:

$$
c_{t}=\frac{C_{t}}{C_{t}^{a}}
$$

\footnotetext{
${ }^{14}$ The decentralization of the private information Pareto optimal allocation exploits in a part a derivation provided to me by Fernando Alvarez.
} 
I divide both sides by $\left(C_{t}^{a}\right)^{-\rho}$ :

$$
\left(c_{t}\right)^{-\rho} E\left[\left(\frac{S_{t+1}}{S_{t}}\right) Q_{t+1} \mid \mathcal{F}_{t}\right]=\exp (-\delta) E\left[\left(c_{t+1}\right)^{-\rho}\left(\frac{C_{t+1}^{a}}{C_{t}^{a}}\right)^{-\rho} Q_{t+1} \mid \mathcal{G}_{t}\right]
$$

I consider two possible ways to represent the stochastic discount factor increment. First divide by the (scaled) marginal utility $\left(c_{t}\right)^{-\rho}$ and apply the Law of Iterated Expectations:

$$
E\left[\left(\frac{S_{t+1}}{S_{t}}\right) Q_{t+1} \mid \mathcal{F}_{t}\right]=\exp (-\delta) E\left[\left(\frac{c_{t+1}}{c_{t}}\right)^{-\rho}\left(\frac{C_{t+1}^{a}}{C_{t}^{a}}\right)^{-\rho} Q_{t+1} \mid \mathcal{F}_{t}\right]
$$

By allowing trades among assets that include any bounded payoff that is $\mathcal{F}_{t+1}$ measurable, it follows that

$$
\frac{S_{t+1}}{S_{t}}=\exp (-\delta)\left(\frac{C_{t+1}^{a}}{C_{t}^{a}}\right)^{-\rho} E\left[\left(\frac{c_{t+1}}{c_{t}}\right)^{-\rho} \mid \mathcal{F}_{t+1}\right]
$$

See Appendix A. This generalizes the usual power utility model representative agent specification of the one-period stochastic discount factor. Because of the preclusion of trading based on idiosyncratic shocks, investors equate the conditional expectations of their intertemporal marginal rates of substitution conditioned only on aggregate shocks. This gives one representation of the limited ability to share risks with this market structure.

For an alternative representation, use the Law of Iterated Expectations on both the left and right-hand sides of (19) to argue that

$$
E\left[\left(c_{t}\right)^{-\rho} \mid \mathcal{F}_{t}\right] E\left[\left(\frac{S_{t+1}}{S_{t}}\right) Q_{t+1} \mid \mathcal{F}_{t}\right]=\exp (-\delta) E\left(E\left[\left(c_{t+1}\right)^{-\rho} \mid \mathcal{F}_{t+1}\right]\left(\frac{C_{t+1}^{a}}{C_{t}^{a}}\right)^{-\rho} Q_{t+1} \mid \mathcal{F}_{t}\right)
$$

Again I use the flexibility to trade based on aggregate shocks to claim that

$$
\frac{S_{t+1}}{S_{t}}=\exp (-\delta)\left(\frac{C_{t+1}^{a}}{C_{t}^{a}}\right)^{-\rho} \frac{E\left[\left(c_{t+1}\right)^{-\rho} \mid \mathcal{F}_{t+1}\right]}{E\left[\left(c_{t}\right)^{-\rho} \mid \mathcal{F}_{t}\right]} .
$$

For specification (18) suggested by Constantinides and Duffie (1996), $c_{t+1}-c_{t}$ is conditionally log-normally distributed and as a consequence,

$$
E\left[\left(\frac{c_{t+1}}{c_{t}}\right)^{-\rho} \mid \mathcal{F}_{t+1}\right]=\exp \left[\frac{\rho(\rho+1)\left(Z_{t+1}\right)^{2}}{2}\right]
$$


In this special case,

$$
\frac{S_{t+1}}{S_{t}}=\exp (-\delta)\left(\frac{C_{t+1}^{a}}{C_{t}^{a}}\right)^{-\rho} \exp \left[\frac{\rho(\rho+1)\left(Z_{t+1}\right)^{2}}{2}\right]
$$

This is just an example, but an informative one. The consumption distribution "fans out" and its dependence on the aggregate state variable $Z_{t+1}$ implies permanent consequences for the the stochastic discount factor. ${ }^{15}$ There are other mechanisms that might well push against the fanning which are abstracted from in this formulation. For instance, overlapping generations models can induce some reversion depending on how the generations are connected and how new generations are endowed.

\subsubsection{Efficient Allocations with Private Information}

One explicit rationale for limiting contracting to aggregate shocks is that idiosyncratic shocks reflect private information. In an interesting contribution, Kocherlakota and Pistaferri $(2007,2009)$ propose a decentralization of constrained efficient allocations represented via the construction of a stochastic discount factor. Kocherlakota and Pistaferri consider the case of constraint efficient allocations where agents' preferences are given by expected discounted utility with an additive sub-utility of consumption and leisure (or effort), and where the consumption sub-utility is specified as a power utility function, $\rho=\gamma$. Individual agents' leisure (effort) needed to produce a given output is private information. Individuals cannot hide consumption, however, through even inefficient storage.

Kocherlakota and Pistaferri (2009) take as given the solution of planning problem where agents effort is unobservable. How this efficient allocation is attained is an interesting question in its own right, a question that is of direct interest and discussed extensively in the literature on contracting in the presence of private information. To decentralize these allocations, Kocherlakota and Pistaferri consider intermediaries that can observe the consumption of the agents and that can trade among themselves. They distinguish between aggregate shocks (which are public) and idiosyncratic shocks which are private but diversifiable as with the incomplete financial market model that I discussed previously. Intermediaries trade among themselves in complete markets on all public shocks and engage

\footnotetext{
${ }^{15}$ In the degenerate case in which $Z$ is constant over time, the impact of the cross-sectional distribution will only be to scale the stochastic discount factor and hence prices will be scaled by a common factor. Risk and shock price elasticities will coincide with those from the corresponding representative consumer model.
} 
a large number of agents so they diversify completely the privately observed shocks. The contract of the intermediary with the agents ensures that the reports are correct. The objective of the intermediaries is to minimize the cost, at market prices, of delivering agents a given lifetime utility. This intermediary provides a way to deduce the corresponding stochastic discount factor for assigning values to payoffs on the aggregate state.

I introduce a random variable $Q_{t+1}$ that is $\mathcal{F}_{t+1}$ measurable. Imagine adding $\mathrm{r} Q_{t+1}$ to the $t+1$ period utilities instead to the period $t+1$ consumption. Due to the additive separability of the period utility function adding an amount of utils both on $t$ and across all continuations at $t+1$ does change the incentives for the choice of leisure (effort). This leads me to consider the equivalent adjustment $\Delta_{t+1}(r)$ to consumption:

$$
\mathrm{r} Q_{t+1}+U\left(C_{t+1}\right)=U\left[C_{t+1}+\Delta_{t+1}(\mathrm{r})\right]
$$

I have altered the $t+1$ period cross-sectional utility in a way that is equivalent to changing the utility to the efficient allocation of consumption in the cross sectional distribution at date $t+1$ in a manner that does not depend on the idiosyncratic shocks. To support this change, however, the change in consumption $\Delta_{t+1}(r)$ does depend on idiosyncratic shocks. Differentiating with respect to $r$ :

$$
Q_{t+1}=\left.\left(C_{t+1}\right)^{-\rho} \frac{\Delta_{t+1}}{d r}\right|_{r=0} .
$$

Thus

$$
\left.\frac{d \Delta_{t+1}}{d r}\right|_{r=0}=Q_{t+1}\left(C_{t+1}\right)^{\rho} .
$$

To compensate for the $Q_{t+1}$ change in the next period (date $t+1$ ) utility, subtract

$$
\exp (-\delta) E\left(Q_{t+1} \mid \mathcal{G}_{t}\right)=\exp (-\delta) E\left(Q_{t+1} \mid \mathcal{G}_{t}\right)
$$

from the current (date $t$ ) utility. This leads me to solve:

$$
-\mathrm{r} \exp (-\delta) E\left(Q_{t+1} \mid \mathcal{F}_{t}\right)+U\left(C_{t}\right)=U\left[C_{t}-\Theta_{t}(\mathrm{r})\right]
$$

Again differentiating with respect to $r$,

$$
-\exp (-\delta) E\left(Q_{t+1} \mid \mathcal{F}_{t}\right)=\left.\left(C_{t}\right)^{-\rho} \frac{d \Theta_{t}}{d \boldsymbol{r}}\right|_{r=0},
$$


or

$$
\left.\frac{d \Theta_{t}}{d r}\right|_{r=0}=\exp (-\delta) E\left(Q_{t+1} \mid \mathcal{F}_{t}\right)\left(C_{t}\right)^{\rho}
$$

The members of our family of $r Q_{t+1}$ perturbations have the same continuation values as those in the efficient allocation. By design the perturbations are equivalent to a transfer of utility across time periods that does not depend on idiosyncratic shocks. These two calculations are inputs into first-order conditions for the financial intermediary.

The financial intermediary solves a cost minimization problem:

$$
\min _{r}-E\left[\Theta_{t}(\mathrm{r}) \mid \mathcal{F}_{t}\right]+E\left[\left(\frac{S_{t+1}}{S_{t}}\right) \Delta_{t+1}(\mathrm{r}) \mid \mathcal{F}_{t}\right]
$$

We want the minimizing solution to occur when $r$ is set to zero. The first-order conditions are:

$$
-E\left[\left.\frac{d \Theta_{t}}{d r}\right|_{r=0} \mid \mathcal{F}_{t}\right]+E\left[\left.\left(\frac{S_{t+1}}{S_{t}}\right) \frac{d \Delta_{t+1}}{d r}\right|_{r=0} \mid \mathcal{F}_{t}\right]=0
$$

Substituting for the $\Delta_{t}$ and $\Theta_{t}$ derivatives,

$$
-\exp (-\delta) E\left(Q_{t+1} \mid \mathcal{F}_{t}\right) E\left[\left(C_{t}\right)^{\rho} \mid \mathcal{F}_{t}\right]+E\left[\left(\frac{S_{t+1}}{S_{t}}\right) Q_{t+1} E\left[\left(C_{t+1}\right)^{\rho} \mid \mathcal{F}_{t+1}\right] \mid \mathcal{F}_{t}\right]=0
$$

Let

$$
D_{t+1}=\exp (\delta)\left(\frac{S_{t+1}}{S_{t}}\right) \frac{\left(C_{t+1}^{a}\right)^{\rho} E\left[\left(c_{t+1}\right)^{\rho} \mid \mathcal{F}_{t+1}\right]}{\left(C_{t}^{a}\right)^{\rho} E\left[\left(c_{t}\right)^{\rho} \mid \mathcal{F}_{t}\right]}
$$

where I have used the fact that the cross-sectional averages $C_{t}^{a}$ and $C_{t+1}^{a}$ are in the respective information sets of aggregate variables. Then

$$
E\left(Q_{t+1} \mid \mathcal{F}_{t}\right)=E\left[D_{t+1} Q_{t+1} \mid \mathcal{F}_{t}\right]
$$

Given flexibility in the choice of the $\mathcal{F}_{t+1}$ measurable random variable $Q_{t+1}$, I show in Appendix A that $D_{t+1}=1$, giving rise to the "inverse Euler equation:"

$$
\left(\frac{S_{t+1}}{S_{t}}\right)=\exp (-\delta)\left(\frac{C_{t+1}^{a}}{C_{t}^{a}}\right)^{-\rho} \frac{E\left[\left(c_{t}\right)^{\rho} \mid \mathcal{F}_{t}\right]}{E\left[\left(c_{t+1}\right)^{\rho} \mid \mathcal{F}_{t+1}\right]}
$$

suggested by Kocherlakota and Pistaferri (2009). The "inverse" nature of the Euler equation emerges because my use of utility based perturbations based on aggregate shocks rather than direct consumption-based perturbations. This type of Euler equation is familiar since 
the seminal work of Rogerson (1985).

An alternative, but complementary analysis, derives the full solution to the constraint efficient allocation. At least since the work of Atkeson and Lucas (1992), it is known that even temporary idiosyncratic shocks create a persistent trend in dispersion of consumption. Hence this particular way of modeling private information has the potential of important effects the long-term (martingale) component to valuation.

In the incomplete contracting framework we were led to consider the time series of crosssectional moments $\left\{E\left[\left(c_{t}\right)^{-\rho} \mid \mathcal{F}_{t}\right]: t=1,2, \ldots\right\}$ whereas in this private information, Pareto efficient economy we are led to consider $\left\{E\left[\left(c_{t}\right)^{\rho} \mid \mathcal{F}_{t}\right]: t=1,2, \ldots\right\}$. These two models feature rather different attributes, including tails behavior of the cross-sectional distribution for consumption. Both, however, suggest the possibility of long-term contributions to valuation because of the dependence of the cross-sectional distribution on economic aggregates. There are important measurement challenges that arise in exploring the empirical underpinnings of these models, but some valuable initial steps have been taken by Brav et al. (2002), Cogley (2002) and Kocherlakota and Pistaferri (2009).

\subsection{Solvency Constraints}

In this section I discuss the representation of stochastic discount factor in models where agents face occasionally binding solvency constraints. One tractable class of models that features incomplete risk sharing is the one where agents have access to complete markets but where the total value of their financial wealth is constrained (from below) in a state contingent manner. Following Luttmer (1992, 1996) and He and Modest (1995), I refer to such constraints as solvency constriants. In contrast to the models with incomplete contracting based on information constraints, I no longer distinguish between $\mathcal{G}_{t}$ and $\mathcal{F}_{t}$; but I do allow for some ex ante heterogeneity in endowments or labor income. Suppose there are $i$ types of investors, each with consumption $C_{t}^{i}$. Investor types may have different initial asset holdings and may different labor income or endowment processes. Let $C_{t}^{a}$

denote the average across all consumers and $c_{t}^{i}=\frac{C_{t}^{i}}{C_{t}^{a}}$. Under expected discounted utility preferences with a power specification $(\rho=\gamma)$, the stochastic discount factor increment is:

$$
\frac{S_{t+1}}{S_{t}}=\exp (-\delta)\left(\frac{C_{t+1}^{a}}{C_{t}^{a}}\right)^{-\rho} \max _{i}\left\{\left(\frac{c_{t+1}^{i}}{c_{t}^{i}}\right)^{-\rho}\right\} .
$$


To better understand the origin of this formula, notice that an implication of it is:

$$
\frac{S_{t+1}}{S_{t}} \geq \exp (-\delta)\left(\frac{C_{t+1}^{i}}{C_{t}^{i}}\right)^{-\rho}
$$

which is featured in the work Luttmer $(1992,1996)$ and He and Modest (1995).

To understand better this inequality, observe that positive scalar multiples $r \geq 0$ of a positive payoff $Q_{t+1} \geq 0$ when added to composite equilibrium portfolio payoff of person $i$ at date $t+1$ will continue to satisfy the solvency constraint. Thus such a perturbation is an admissible one. When I optimize with respect to $r$, I now impose the constraint that $r \geq 0$; and this introduces a Kuhn-Tucker multiplier into the calculation. The first-order condition for $r$ is

$$
\left(C_{t}^{i}\right)^{-\rho} E\left[\left(\frac{S_{t+1}}{S_{t}}\right) Q_{t+1} \mid \mathcal{F}_{t}\right] \geq \exp (-\delta) E\left[\left(C_{t+1}^{i}\right)^{-\rho} Q_{t+1} \mid \mathcal{F}_{t}\right]
$$

where the inequality is included in case the nonnegativity constraint on $r$ is binding. Since this inequality is true for any bounded, positive nonnegative $\mathcal{F}_{t+1}$ measurable payoff $Q_{t+1}$, inequality relation (25) holds.

Formula (24) is a stronger restriction and follows since equilibrium prices are determined by having at least one individual that is unconstrained in the different realized date $t+1$ states of the world. The max operator captures the feature that the types with the highest valuation are unconstrained. While in principle expression (24) can be estimated using an empirical counterpart to the type's $i$ consumption, the presence of the max using error ridden data makes the measurement daunting.

Luttmer (1992) takes a different approach by exploiting the implications via aggregation of solvency constraints and analyzing the resulting inequality restriction. ${ }^{16}$ This same argument is revealing for my purposes as well. Since $\rho$ is positive, inequality (25) implies that

$$
C_{t}^{i}\left(\frac{S_{t+1}}{S_{t}}\right)^{-\frac{1}{\rho}} \leq \exp (-\delta) C_{t+1}^{i}
$$

where inequality is reversed because $-\frac{1}{\rho}<0$. Forming a cross-sectional average, preserves the inequality:

$$
C_{t}^{a}\left[\exp (\delta) \frac{S_{t+1}}{S_{t}}\right]^{-\frac{1}{\rho}} \leq C_{t+1}^{a}
$$

\footnotetext{
${ }^{16}$ See Hansen et al. (1995) for a discussion of econometric methods that support such an approach.
} 
Raising both sides to the negative power $-\rho$ reverses again the inequality:

$$
\left(C_{t}^{a}\right)^{-\rho}\left[\exp (\delta) \frac{S_{t+1}}{S_{t}}\right] \geq\left(C_{t+1}^{a}\right)^{-\rho}
$$

Rearranging terms gives the inequality of interest

$$
\frac{S_{t+1}}{S_{t}} \geq \exp (-\delta)\left(\frac{C_{t+1}^{a}}{C_{t}^{a}}\right)^{-\rho}
$$

Form this inequality, we can rule out any hope that solvency-constraint models only have transient consequences for valuation because we cannot hope to write

$$
\frac{S_{t+1}}{S_{t}}=\exp (-\delta)\left(\frac{C_{t+1}^{a}}{C_{t}^{a}}\right)^{-\rho}\left[\frac{h\left(X_{t+1}\right)}{h\left(X_{t}\right)}\right]
$$

for some $h$ and some stochastically stable process $X$, unless of course $h(X)$ is constant with probability one. In the degenerate case, the solvency constraints are not binding. At the very least the interest rate rates on discount bonds, including the long-term interest rate, have to be smallar than those in the corresponding representative consumer economy.

Alvarez and Jermann (2000, 2001) and Chien and Lustig (2010) impose more structure on the economic environment in order to get sharper predictions about the consumption allocations. They use limited commitment as a device to set the solvency thresholds needed to compute an equilibrium. ${ }^{17}$ Following Kehoe and Levine (1993), these authors introduce an outside option that becomes operative if an investor defaults on the financial obligations. The threat of the outside option determines the level of the solvency constraint that is imposed in a financial market decentralization. Solvency constraints are chosen to prevent that the utility value to staying in a market risk sharing arrangement to be at least as high as the utility of the corresponding outside options. Alvarez and Jermann (2001) and Chien and Lustig (2010) differ in terms the precise natures of the market exclusions that occur when investor walk away from their financial market obligations. Alvarez and Jermann (2000) argue that the cross-sectional distribution in example economies with solvency constraints stable in the sense that $C_{t}^{i}=h_{i}\left(X_{t}\right) C_{t}^{a}$ for some stochastically stable Markov process $X$.

\footnotetext{
${ }^{17}$ Zhang (1997) considers a related environment in which borrowing constraints are endogenously determined as implication of threats to default. Alvarez and Jermann (2000, 2001) and Chien and Lustig (2010) extend Zhang (1997) by introducing a richer collection of security markets.
} 
Thus

$$
\frac{S_{t+1}}{S_{t}}=\exp (-\delta)\left(\frac{C_{t+1}^{a}}{C_{t}^{a}}\right)^{-\rho} \max _{i}\left\{\left(\frac{h_{i}\left(X_{t+1}\right)}{h_{i}\left(X_{t}\right)}\right)^{-\rho}\right\} .
$$

Notice that the objective of the max operation is ratio of a common function of the Markov state over adjacent periods. Even so, given our previous argument the outcome of this maximization will not have an expression as an analogous ratio. Even with a stable consumption allocation, the presence of solvency constraints justified by limited commitment may have long-term consequences for valuation. ${ }^{18}$

Chien and Lustig (2010) also provide a suggestive characterization for the stochastic discount factor ratio of the form:

$$
\frac{S_{t+1}}{S_{t}}=\exp (-\delta)\left(\frac{C_{t+1}^{a}}{C_{t}^{a}}\right)^{-\rho}\left(\frac{Z_{t+1}}{Z_{t}}\right)^{\rho}
$$

Thus $\left(Z_{t}\right)^{\rho}=S_{t}^{c}$ in representation (16). Chien and Lustig interpret $Z$ and characterize the ratio $\frac{Z_{t+1}}{Z_{t}}$ using numerical methods. In particular, they show that the positive process $Z$ does not decrease over time. ${ }^{19}$ This property for $Z$ is to be anticipated from (26). Unless it is degenerate, such a process cannot be stationary; but its logarithm can have stationary increments as presumed in my analysis.

Even though the consumption distribution may not "fan out" over time, evidently the introduction of solvency constraints has important long-term consequences for valuation. A remaining challenge is to understand better when the implied state dependence in the cross-sectional distribution of individual consumption ratios induces changes in the long-

\footnotetext{
${ }^{18}$ Alvarez and Jermann (2000) derive a different type of factorization of a stochastic discount factor under some very special restrictions. They express the endowments for each investor type as a product of the aggregate endowment and a share of that endowment. They suppose that the growth in the aggregate endowment is itself independent and identically distributed and that the aggregate endowment process is independent of the vector of endowment share processes. They argue that equilibrium stochastic discount factor is the product of the corresponding representative consumer stochastic discount factor and a term that is independent of the process for aggregate endowment growth. (See the proof of their Proposition 5.4.) Thus the two terms in factorization (16) are statistically independent in their example. The prices of payoffs that depend only on aggregate endowment growth process over fixed investment horizon have common term that emerges because of the contribution of the share process history to the stochastic discount factor. By forming price ratios, this share-process contribution to valuation is netted out. For a cash flow $G$ that depends only on the aggregate endowment and not on the share process, the cash-flow risk premia as measured by formula (15) will be the same as for the corresponding representative consumer model with iid consumption growth. An analogous simplification applies to payoffs that depend only on share process and not on the aggregate endowment.

${ }^{19}$ See also Alvarez and Jermann (2000) Proposition 5.2 for the analogous result for the limited commitment economies that they study.
} 
term risk prices.

\subsection{Segmented Market and Nominal Shocks}

In this section I explore environments that feature both nominal shocks and segmented asset markets. The transient consequences of nominal shocks has been featured in other environments. For instance in log-linear specifications of the macro time series, Blanchard and Quah (1989) and Shapiro and Watson (1988) use the transient nature of nominal shocks as a device to identify tansient and permanent sources of economic fluctuations. Here I explore economic models with explicit transition mechanisms to investigate further the transient nature of nominal shocks for valuation including adjustments for risk exposure.

As an illustration, I consider the stylized model of Alvarez et al. (2002) and Alvarez et al. (2009) where both nominal cash-flows and segmentation are introduced. For simplicity, these models presume a binding cash-in-advance constraint, and hence the price level is proportional to money supply. Consumers can transfer cash between their "brokerage account" -where an intermediary with access to complete security markets manages their portfolio- and a liquid asset that must be use for consumption expenditures. For consumers to embark on this transfer, they must pay a fixed cost. If they decide not to pay the fixed cost, they must consume the real value of the accumulated nominal income. In equilibrium only some of the consumers participate in asset markets, but those that do so have the same consumption, which I denote by $C_{t}^{p}$. In this case the one-period stochastic discount factor is given by the corresponding one-period intertemporal marginal rate of substitution of the participants adjusted for changes in the nominal price level

$$
\frac{S_{t+1}}{S_{t}}=\exp (-\delta)\left(\frac{P_{t}}{P_{t+1}}\right)\left(\frac{C_{t+1}^{p}}{C_{t}^{p}}\right)^{-\rho}
$$

where $P_{t}$ is the date $t$ price level and where $S_{t}$ is the nominal discount factor. This formula is true even though, the identity of the participants changes over time. The equilibrium of this model is such that

$$
C_{t}^{p}=C_{t}^{a} h_{p}\left(\log m_{t}-\log m_{t-1}\right)
$$

where $\log m_{t}-\log m_{t-1}$ is the growth rate of the money supply and $C_{t}^{a}$ is aggregate consumption. Thus

$$
\frac{S_{t+1}}{S_{t}}=\exp (-\delta)\left(\frac{P_{t}}{P_{t+1}}\right)\left(\frac{C_{t+1}^{a}}{C_{t}^{a}}\right)^{-\rho}\left[\frac{h\left(\log m_{t+1}-\log m_{t}\right)}{h\left(\log m_{t}-\log m_{t-1}\right)}\right]
$$


where $h=\left(h_{p}\right)^{-\rho}$.

In transaction cost models such as this one, the impact on risk pricing is transient relative to a standard power utility model. The impact of changes in participation is captured by the function $h$ of the presumed stationary growth rate in the money supply. Nominal fluctuations influence real outcomes The factorization in (27) features both standard nominal effects on valuation from both the nominal price level $P$, and aggregate consumption $C^{a}$. Both contribute to the martingale component of the stochastic discount factor $S$. This type of models can and has been used to study both term premium for nominal interest rates as well for nominal exchange rates. Third, one can use the growth decomposition described in equation (13) by letting $G=P$ to distinguish real cash flows from nominal ones or more generally by letting a multiplicative component of $G$ be $P$ as a device to take nominal growth into account for computing real risk-price and shock price-elasticities induced by nominal shocks.

In this section I have only "scratched the surface" so to speak on characterizing how and when market restrictions alter the term structure of shock an risk-price elasticities through it implications for the time series behavior of cross-sectional distributions. As a starting point I have examined when the long-term discount rate and the martingale components of stochastic discount factors are altered, but as I argued earlier in a different context this is merely a starting point for a more complete analysis. 


\section{Conclusions}

In this chapter I have focused on characterizing asset values through the lens of economic models of valuation. By using structural models, models with an explicitly specified preference and market structure, researchers can assign values to a rich collection of cash flows and risk prices for the exposures to alternative underlying macroeconomic shocks. These DVD methods that I discussed allow researchers to extract pricing implications for cash flows without resort to log-linearization. I consider the global "entropy" methods and local "elasticity" methods based on perturbing the exposure of cash flows to shocks as complementary devices to characterized the sensitivity of risk prices to the investment horizon. The DVD methods are supported in part by a factorization that provides a mathematical formalization of permanent and transitory components to valuation. The distinction between permanent and transitory rests formally on limiting behavior when we extend the investment horizon. As with related time series decompositions, this distinction enhances both model building and testing by clarifying when the permanent and transitory distinction is sharp and when it is blurred.

While this is a chapter about models, it also suggestive of what hypothetical securities might be most useful in distinguishing among competing models. As time series data on richer collections of equity-based derivative contracts become available, they offer the promise to pose direct challenges to the underlying pricing of cash flows. Complementary econometric and empirical advances will enhance our understanding of the empirical underpinnings of structural models of asset prices. 


\section{A Limited contracting economies revisited}

In this appendix I complete two of the arguments made in Section 4.1. Consider first an argument in 4.1.1. There I showed that

$$
E\left[\left(\frac{S_{t+1}}{S_{t}}\right) Q_{t+1} \mid \mathcal{F}_{t}\right]=\exp (-\delta) E\left[\left(\frac{c_{t+1}}{c_{t}}\right)^{-\rho}\left(\frac{C_{t+1}^{a}}{C_{t}^{a}}\right)^{-\rho} Q_{t+1} \mid \mathcal{F}_{t}\right]
$$

My aim is to show that formula (20) is satisfied. For convenience, I rewrite it:

$$
\frac{S_{t+1}}{S_{t}}=\exp (-\delta)\left(\frac{C_{t+1}^{a}}{C_{t}^{a}}\right)^{-\rho} E\left[\left(\frac{c_{t+1}}{c_{t}}\right)^{-\rho} \mid \mathcal{F}_{t+1}\right]
$$

Let

$$
Q_{t+1}=\left\{\begin{array}{cc}
1 & \left(\frac{S_{t+1}}{S_{t}}\right)>\exp (-\delta)\left(\frac{C_{t+1}^{a}}{C_{t}^{a}}\right)^{-\rho} E\left[\left(\frac{c_{t+1}}{c_{t}}\right)^{-\rho} \mid \mathcal{F}_{t+1}\right] \\
0 & \text { otherwise }
\end{array}\right.
$$

Then it follows from (28) that

$$
\left(\frac{S_{t+1}}{S_{t}}\right)>\exp (-\delta)\left(\frac{C_{t+1}^{a}}{C_{t}^{a}}\right)^{-\rho} E\left[\left(\frac{c_{t+1}}{c_{t}}\right)^{-\rho} \mid \mathcal{F}_{t+1}\right]
$$

with probability zero. An entirely similar argument implies the reverse inequality. Thus representation (20) is valid. Since a similar argument proves (21), I do not repeat the logic.

Consider next an argument in Section 4.1.2 Recall the construction:

$$
D_{t+1}=\left(\frac{S_{t+1}}{S_{t}}\right) \frac{\exp (\delta) E\left[\left(C_{t+1}\right)^{\rho} \mid \mathcal{F}_{t+1}\right]}{E\left[\left(C_{t}\right)^{\rho} \mid \mathcal{F}_{t}\right]}
$$

and the implication

$$
E\left(D_{t+1} Q_{t+1} \mid \mathcal{F}_{t}\right)=E\left(Q_{t+1} \mid \mathcal{F}_{t}\right) .
$$

I now show that by exploiting the flexibility in the choice of $Z_{t+1}, D_{t+1}=1$ giving rise to the inverse Euler equation: (23) Let

$$
Q_{t+1}=\left\{\begin{array}{cc}
D_{t+1} & \text { if } \mathrm{b}>D_{t+1}>1 \\
0 & \text { otherwise }
\end{array}\right.
$$

I impose an upper bound on $Q_{t+1}$ to ensure that perturbation in the date $t+1$ utility can be 
implemented by choices of individual consumptions. It follows that $\operatorname{Pr}\left\{\mathrm{b} \geq Q_{t+1}>1\right\}=0$. Since $\mathbf{b}$ is arbitrary, it must also be true that $\operatorname{Pr}\left\{D_{t+1}>1\right\}=0$. Similarly, form

$$
Q_{t+1}=\left\{\begin{array}{cc}
0 & \text { if } D_{t+1} \geq 1 \\
D_{t} & \text { otherwise }
\end{array} .\right.
$$

It then follows that $\operatorname{Pr}\left\{D_{t+1}<1\right\}=0$. Thus $D_{t+1}=1$. 


\section{References}

Abel, Andrew B. 1990. Asset Prices under Habit Formation and Catching up with the Joneses. The American Economic Review 80 (2):pp. 38-42.

- 2002. An exploration of the effects of pessimism and doubt on asset returns. Journal of Economic Dynamics and Control 26.

Ai, Hengjie, Mariano Massimiliano Croce, and Kai Li. 2010. Toward a Quantitative General Equilibrium Asset Pricing Model with Intangible Capital. Mimeo.

Alvarez, F., A. Atkeson, and P.J. Kehoe. 2002. Money, Interest Rates, and Exchange Rates with Endogenously Segmented Asset Market. Journal of Political Economy 1 (110):773112.

- 2009. Time-Varying Risk, Interest Rates, and Exchange Rates in General Equilibrium. Review of Economic Studies 76 (3):851-878.

Alvarez, Fernando and Urban Jermann. 2000. Efficiency, Equilibrium, and Asset Pricing with Risk of Default. Econometrica 775-798.

Alvarez, Fernando and Urban J. Jermann. 2001. Quantitative Asset Pricing Implications of Endogenous Solvency Constraints. The Review of Financial Studies 14 (4):pp. 11171151.

- 2005. Using Asset Prices to Measure the Persistence of the Marginal Utility of Wealth. Econometrica 73 (6):pp. 1977-2016.

Anderson, Evan W., Lars Peter Hansen, and Thomas J. Sargent. 2003. A Quartet of Semigroups for Model Specification, Robustness, Prices of Risk, and Model Detection. Journal of the European Economic Association 1 (1):68-123.

Atkeson, A. and R.E. Jr. Lucas. 1992. On efficient distribution with private information. The Review of Economic Studies 59 (3):427-453.

Backus, David, Mikhail Chernov, and Stanley Zin. 2011. Sources of Entropy in Representative Agent Models. Unpublished working paper.

Bansal, Ravi and Bruce N. Lehmann. 1997. Growth-optimal Portfolio Restrictions on Asset Pricing Models. Macroeconomic Dynamics 1 (2):333-354. 
Bansal, Ravi and Amir Yaron. 2004. Risks for the Long Run: A Potential Resolution of Asset Pricing Puzzles. Journal of Finance 59 (4):1481-1509.

Bickel, Peter J., Ya'acov Ritov, and Tobias Ryden. 1998. Asymptotic Normality of the Maximum-Likelihood Estimator for General Hidden Markov Models. The Annals of Statistics 26 (4):1614-1635.

van Binsbergen, Jules, Michael Brandt, and Ralph Koijen. 2011. On the Timing and Pricing of Dividends. American Economic Review forthcoming.

van Binsbergen, Jules, Michael Brandt, Ralph Koijen, and Evert Vrugt. 2012. Equity Yields. Becker-Friedman Institute Working Paper.

Blanchard, Olivier Jean and Danny Quah. 1989. The Dynamic Effects of Aggregate Demand and Supply Disturbances. The American Economic Review 79 (4):pp. 655-673.

Borovicka, Jaroslav and Lars Peter Hansen. 2012. Examining macroeconomic models through the lens of asset pricing. Working Paper Series WP-2012-01, Federal Reserve Bank of Chicago.

Borovička, Jaroslav, Lars Peter Hansen, Mark Hendricks, and José A. Scheinkman. 2011. Risk Price Dynamics. Journal of Financial Econometrics 9:3-65.

Brav, Alon, George M. Constantinides, and Christopher C. Geczy. 2002. Asset Pricing with Heterogeneous Consumers and Limited Participation: Empirical Evidence. Journal of Political Economy 110 (4):pp. 793-824.

Campbell, John Y. and John H. Cochrane. 1999. By Force of Habit. Journal of Political Economy 107 (2):205-251.

Cecchetti, Stephen G., Pok-sang Lam, and Nelson C. Mark. 2000. Asset Pricing with Distorted Beliefs: Are Equity Returns Too Good to Be True? The American Economic Review 90 (4):pp. 787-805.

Chen, Zengjing and Larry Epstein. 2002. Ambiguity, Risk, and Asset Returns in Continuous Time. Econometrica 70 (4):1403-1443.

Chien, YiLi and Hanno Lustig. 2010. The Market Price of Aggregate Risk and the Wealth Distribution. Review of Financial Studies 23 (4):1596-1650. 
Cochrane, John H. 2001. Asset Pricing. Princeton, N.J.: Princeton University Press.

Cogley, Timothy. 2002. Idiosyncratic risk and the equity premium: evidence from the consumer expenditure survey. Journal of Monetary Economics 49 (2):309 - 334.

Constantinides, George M. 1990. Habit Formation: A Resolution of the Equity Premium Puzzle. Journal of Political Economy 98 (3):519-43.

Constantinides, George M. and Darrell Duffie. 1996. Asset Pricing with Heterogeneous Agents. Journal of Political Economy 104 (2):219-240.

Dybvig, Philip H., Jr. Ingersoll, Jonathan E., and Stephen A. Ross. 1996. Long Forward and Zero-Coupon Rates Can Never Fall. The Journal of Business 69 (1).

Epstein, L. and S. Zin. 1989. Substitution, Risk Aversion and the Temporal Behavior of Consumption and Asset Returns: A Theoretical Framework. Econometrica 57 (4):937969.

Garcia, Rene, Eric Renault, and Andrei Semenov. 2006. Disentangling risk aversion and intertemporal substitution through a reference level. Finance Research Letters 3:181-193.

Granger, C. W. J. 1969. Investigating Causal Relations by Econometric Models with Crossspectral Methods. Econometrica 37:424-438.

Hansen, Lars Peter. 2012. Dynamic Valuation Decomposition within Stochastic Economies. Econometrica 911-967 (3). Fisher-Schultz Lecture at the European Meetings of the Econometric Society.

Hansen, Lars Peter and Ravi Jagannathan. 1991. Implications of Security Market Data for Models of Dynamic Economies. Journal of Political Economy 99:225-262.

Hansen, Lars Peter and Scott F. Richard. 1987. The Role of Conditioning Information in Deducing Testable Restrictions Implied by Dynamic Asset Pricing Models. Econometrica 50:587-614.

Hansen, Lars Peter and José A. Scheinkman. 2009. Long-Term Risk: An Operator Approach. Econometrica 77:177-234.

—. 2012a. Pricing Growth-Rate Risk. Finance and Stochastics 16 (1):1-15. 
- 2012b. Recursive utility in a Markov environment with stochastic growth. Proceedings of the National Academy of Sciences forthcoming.

Hansen, Lars Peter, John Heaton, and Erzo G. J. Luttmer. 1995. Econometric Evaluation of Asset Pricing Models. The Review of Financial Studies 8 (2):pp. 237-274.

Hansen, Lars Peter, Thomas J. Sargent, and Jr. Tallarini, Thomas D. 1999. Robust Permanent Income and Pricing. The Review of Economic Studies 66 (4):873-907.

Hansen, Lars Peter, John C. Heaton, Junghoon Lee, and Nikolai Roussanov. 2007. Intertemporal Substitution and Risk Aversion. In Handbook of Econometrics, vol 6A, edited by James J. Heckman and Edward E. Leamer. Amsterdam: North-Holland.

Hansen, Lars Peter, John C. Heaton, and Nan Li. 2008. Consumption Strikes Back?: Measuring Long-Run Risk. Journal of Political Economy 116:260-302.

He, Hua and David M. Modest. 1995. Market Frictions and Consumption-Based Asset Pricing. Journal of Political Economy 103 (1):pp. 94-117.

Heaton, John. 1995. An Empirical Investigation of Asset Pricing with Temporally Dependent Preference Specifications. Econometrica 63 (3):681-717.

Ilut, Cosmin L. and Martin Schneider. 2012. Ambiguous Business Cycles. ERID Working Paper No. 123.

Jamshidian, Farshid. 1989. An Exact Bond Option Formula. The Journal of Finance 44 (1):pp. 205-209.

Kehoe, T.J. and D.K. Levine. 1993. Debt-constrained asset markets. The Review of Economic Studies 60 (4):865-888.

Kocherlakota, N. and L. Pistaferri. 2009. Asset pricing implications of Pareto optimality with private information. Journal of Political Economy 117 (3):555-590.

Kocherlakota, N.R. and L. Pistaferri. 2007. Household Heterogeneity and Real Exchange Rates*. The Economic Journal 117 (519):C1-C25.

Kreps, David M. and Evan L. Porteus. 1978. Temporal Resolution of Uncertainty and Dynamic Choice. Econometrica 46 (1):185-200. 
Lettau, Martin and Jessica Wachter. 2007. Why is Long-Horizon Equity Less Risky? A Duration-Based Explanation of the Value Premium. Journal of Finance 62:55-92.

Luttmer, Erzo G. J. 1992. Asset Pricing in Economies with Frictions. Ph.D. thesis, University of Chicago.

- 1996. Asset Pricing in Economies with Frictions. Econometrica 64 (6):1439-67.

Maenhout, Pascal J. 2004. Robust Portfolio Rules and Asset Pricing. Review of Financial Studies 17 (4):951-983.

Menzly, Lior, Tano Santos, and Pietro Veronesi. 2004. Understanding Predictability. Journal of Political Economy 112 (1):1-47.

Meyn, Sean P. and Richard L. Tweedie. 1993. Stability of Markovian Processes III: FosterLyapunov Criteria for Continuous Time Processes. Advances in Applied Probability 25:518-548.

Rogerson, William P. 1985. Repeated Moral Hazard. Econometrica 53 (1).

Rubinstein, Mark. 1976. The Valuation of Uncertain Income Streams and the Pricing of Options. The Bell Journal of Economics 7:407-425.

Santos, Tano and Pietro Veronesi. 2006. Habit Formation, the Cross Section of Stock Returns and the Cash-Flow Risk Puzzle. Columbia University and the University of Chicago.

Shapiro, Matthew D. and Mark W. Watson. 1988. Sources of Business Cycle Fluctuations. NBER Macroeconomics Annual 3:pp. 111-148.

Sims, Christopher A. 1972. Money, Income, and Causality. The American Economic Review 62 (4):pp. 540-552.

Singleton, Kenneth J. 2006. Empirical Dynamic Asset Pricing: Model Specification and Econometric Assessment. Priceton, N.J.: Princeton University Press.

Sundaresan, S. M. 1989. Intertemporally dependent preferences and the volatility of consumption and wealth. Review of Financial Studies 2 (1):73-89.

Zhang, Harold H. 1997. Endogenous Borrowing Constraints with Incomplete Markets. The Journal of Finance 52 (5):pp. 2187-2209. 